\title{
Ipsilateral-Dominant Control of Limb Movements in Rodent Posterior Parietal Cortex
}

\author{
(D) Shogo Soma, ${ }^{1,3,4}$ 일 \\ Alain Ríos, ${ }^{2}$ Masanori Kawabata, ${ }^{2}$ Kazuto Kobayashi, ${ }^{5}{ }^{-}$Fusao Kato, ${ }^{6}$ Yutaka Sakai, ${ }^{1,2,3}$ and Yoshikazu Isomura ${ }^{1,2,3}$ \\ ${ }^{1}$ Brain Science Institute and ${ }^{2}$ Graduate School of Brain Sciences, Tamagawa University, Tokyo 194-8610, Japan, ${ }^{3}$ Brain/MINDS, Tokyo, 100-0004, Japan, ${ }^{4}$ Japan \\ Society for the Promotion of Science, Tokyo 102-0083, Japan, ${ }^{5}$ Department of Molecular Genetics, Institute of Biomedical Sciences, Fukushima Medical University \\ School of Medicine, Fukushima 960-1295, Japan, and ' $D$ Department of Neuroscience, The Jikei University School of Medicine, Tokyo 105-8461, Japan
}

It is well known that the posterior parietal cortex (PPC) and frontal motor cortices in primates preferentially control voluntary movements of contralateral limbs. The PPC of rats has been defined based on patterns of thalamic and cortical connectivity. The anatomical characteristics of this area suggest that it may be homologous to the PPC of primates. However, its functional roles in voluntary forelimb movements have not been well understood, particularly in the lateralization of motor limb representation; that is, the limb-specific activity representations for right and left forelimb movements. We examined functional spike activity of the PPC and two motor cortices, the primary motor cortex (M1) and the secondary motor cortex (M2), when head-fixed male rats performed right or left unilateral movements. Unlike primates, PPC neurons in rodents were found to preferentially represent ipsilateral forelimb movements, in contrast to the contralateral preference of M1 and M2 neurons. Consistent with these observations, optogenetic activation of PPC and motor cortices, respectively, evoked ipsilaterally and contralaterally biased forelimb movements. Finally, we examined the effects of optogenetic manipulation on task performance. PPC or M1 inhibition by optogenetic GABA release shifted the behavioral limb preference contralaterally or ipsilaterally, respectively. In addition, weak optogenetic PPC activation, which was insufficient to evoke motor responses by itself, shifted the preference ipsilaterally; although similar M1 activation showed no effects on task performance. These paradoxical observations suggest that the PPC plays evolutionarily different roles in forelimb control between primates and rodents.

Key words: association cortex; channelrhodopsin; limb specificity; optogenetic activation; optogenetic inhibition; transgenic rat

\section{Significance Statement}

In rodents, the primary and secondary motor cortices (M1 and M2, respectively) are involved in voluntary movements with contralateral preference. However, it remains unclear whether and how the posterior parietal cortex (PPC) participates in controlling multiple limb movements. We recorded functional activity from these areas using a behavioral task to monitor movements of the right and left forelimbs separately. PPC neurons preferentially represented ipsilateral forelimb movements and optogenetic PPC activation evoked ipsilaterally biased forelimb movements. Optogenetic PPC inhibition via GABA release shifted the behavioral limb preference contralaterally during task performance, whereas weak optogenetic PPC activation, which was insufficient to evoke motor responses by itself, shifted the preference ipsilaterally. Our findings suggest rodent PPC contributes to ipsilaterally biased motor response and/or planning.

\section{Introduction}

Mammals have functionally differentiated motor-related areas in the frontal part of the cerebral cortex. The primary motor cortex

Received June 14, 2018; revised Nov. 12, 2018; accepted Nov. 18, 2018.

Author contributions: S.S. and Y.I. wrote the first draft of the paper; S.S., F.K., Y.S., and Y.I. edited the paper; S.S., J.Y., and Y.I. designed research; S.S., J.Y., Y.T., S.N., Y.K.S., A.R., M.K., and F.K. performed research; S.S., S.K., Y.T., K.K., and F.K. contributed unpublished reagents/analytic tools; S.S., Y.T., Y.K.S., F.K., and Y.S. analyzed data; S.S. and Y.I. wrote the paper.

This work was supported by Grants-in-Aid for JSPS Research Fellow (Grant JP15J00807 to S.S. and Grant JP $16 J 11697$ to J.Y.); Brain/MINDS (Grant JP18dm0207043 to Y.I.) from AMED, Grants-in-Aid for Scientific Research on Innovative Areas (JP16H01516 to Y.S.; Grant JP26112005 to Y.S. and Y.I.; and Grant JP16H06276 to Y.I.); the Sup-
(M1) is believed to shape concrete motor information to control voluntary movements of contralateral body parts (for primates,

ported Program for the Strategic Research Foundation at Private Universities (Grant S1311009 to F.K. and Grant S1311013 to Y.S. and Y.I.) from MEXT; and CREST (Grant JPMJCR1751 to Y.I.) from JST. We thank Drs. Y. Kawaguchi, M. Kimura, A. Nambu, E. Pastalkova, A. Saiki, and H. Yawo for helpful suggestions and discussions and M. Goto, C. Soai, and H. Yoshimatsu for technical assistance.

The authors declare no competing financial interests.

Correspondence should be addressed to Dr. Shogo Soma, Brain Science Institute, Tamagawa University, 6-1-1

Tamagawa-gakuen, Machida, Tokyo 194-8610, Japan. E-mail: s.soma@lab.tamagawa.ac.jp.

https://doi.org/10.1523/JNEUROSCI.1584-18.2018

Copyright $\odot 2019$ the authors $\quad 0270-6474 / 19 / 390485-18 \$ 15.00 / 0$ 
see Evarts, 1966; Tanji et al., 1987; Griffin et al., 2015; for rodents, see Soma et al., 2017). Higher-order motor areas include the premotor cortex (PM) and supplementary motor area (SMA) in primates and the secondary motor cortex (M2) in rodents. These areas contribute to processing abstract motor information such as motor planning and preparation (for primates, see Weinrich and Wise, 1982; Mushiake et al., 1991; Hoshi and Tanji, 2000; Churchland et al., 2006; for rodents, see Narayanan and Laubach, 2009; Saiki et al., 2014; Li et al., 2016). The majority of primate PM/SMA neurons and rodent M2 neurons represent motor information of bilateral body parts, usually with contralateral bias (for PM, see Kurata, 2010; for SMA, see Nakayama et al., 2015; for M2, see Soma et al., 2017).

The posterior parietal cortex (PPC) in primates also participates in processing motor-related information. For example, the parietal reach region, a subarea of the primate PPC, is involved in forelimb reaching (Taira et al., 1990; Desmurget et al., 1999; Connolly et al., 2003) and the anterior intraparietal area, another PPC subarea, is involved in hand grasping (Taira et al., 1990; Gallese et al., 1994; Sakata et al., 1995). As is the case with the frontal higherorder motor areas (Tanji et al., 1987; Cisek et al., 2003; Kurata, 2007, 2010; Nakayama et al., 2015), primate PPC neurons show contralaterally biased bilateral preference during contralateral and ipsilateral limb movements (Kermadi et al., 2000; Chang et al., 2008; Chang and Snyder, 2012). The bilateral preference of the PPC is also supported by a human brain-imaging study (Stark and Zohary, 2008).

Rodent PPC neurons also display motor-related activation during limb locomotion (Chen et al., 1994; McNaughton et al., 1994; Nitz, 2006; Whitlock et al., 2012; Wilber et al., 2014a). However, the preference for contralateral or ipsilateral limb movements is unresolved because measuring independent movements of individual limbs in a standard freely moving condition is technically difficult (Soma et al., 2014). Recently, we established a novel behavioral task in which rats repeated discrete forelimb movements in a steady posture and demonstrated contralateral and bilateral contribution of $\mathrm{M} 1$ and $\mathrm{M} 2$ neurons, respectively (Soma et al., 2017). In the present study, we investigated the limb preference of rat PPC neurons compared with M1 and M2 neurons using the same behavioral task. Furthermore, we examined the behavioral effects of optogenetic manipulation of PPC activity on contralateral and ipsilateral forelimb movements.

\section{Materials and Methods}

Animals. All experiments were approved by the Animal Research Ethics Committee of Tamagawa University (animal experiment protocol H22/ 27-32) and were performed in accordance with the Fundamental Guidelines for Proper Conduct of Animal Experiment and Related Activities in Academic Research Institutions (Ministry of Education, Culture, Sports, Science, and Technology of Japan) and the Guidelines for Animal Experimentation in Neuroscience (Japan Neuroscience Society). All surgical procedures were performed under appropriate isoflurane anesthesia (see below) and all efforts were made to minimize suffering. Our procedures for animal experiments were established in our previous studies (Isomura et al., 2009; Saiki et al., 2014, 2018; Kimura et al., 2017; Soma et al., 2017; Nonomura et al., 2018). For ex vivo electrophysiology using acute brain slices, the protocol for animal experiments was reviewed and approved by the Institutional Animal Care and Use Committee of Jikei University (No. 2015-070C8) and conformed to the Guidelines for the Proper Conduct of Animal Experiments of the Science Council of Japan (2006).

This study is based on data from three transgenic rat lines. The channelrhodopsin-2 (ChR2)-expressing (Thy1-ChR2) transgenic rats (W-TChR2V4; $n=39$ rats, male, $259 \pm 34 \mathrm{~g}$; Figs. 1, 2, 3, 4, 5, 6, 7, 8, and 10) expressed ChR2-Venus conjugate under the control of the Thy1.2 promoter in cortical and other neurons (Tomita et al., 2009). The vesicular GABA transporter (VGAT)-Cre rats [W-Tg(Slc32a1-cre)3_5Fusa, ID: NBRP-0839; $n=7$ rats, male, $300 \pm 12$ g; Fig. 9B-H] expressed Cre recombinase under the control of the VGAT promoter specifically in inhibitory GABAergic neurons (Igarashi et al., 2018). The VGAT-Cre rats were developed by F. Kato and A. M. Watabe (Jikei University, Japan) with a support from the C.B.S.N project conducted by K. Kobayashi and Y. Yanagawa (Gumma University, Japan) using a VGAT-Cre BAC construct developed by S. Itohara (RIKEN, Japan). For histological confirmation, a VGAT-Cre female rat was crossed with a tdTomato reporter male rat [LE-Tg(Gt(ROSA)26SorCAG-tdTomato)24Jfhy, ID: NBRP-0734; Igarashi et al., 2016] and the resulting pups were used ( $n=$ 2 rats, male, $373 \pm 81$ g; Fig. $9 A$ ). These animals were kept in their home cage under an inverted light schedule (lights off at 9:00 A.M.; lights on at 9:00 P.M.).

Viral vector preparation. We combined the VGAT-Cre rats with adenoassociated viral (AAV) vector serotype 2 (based on the AAV Helper-Free system; Agilent Technologies) to locally express ChR-wide receiver (ChRWR)-Venus in GABAergic neurons in the presence of Cre recombinase (AAV-CAGGS-DIO-ChRWR-Venus vector). The transfer plasmid contained the CAGGS promoter and ChRWR-Venus gene connected to woodchuck hepatitis virus posttranscriptional regulatory element and SV40 polyadenylation signal, in which its expression was at first blocked with the double-floxed inverted orientation (DIO) system. The transfer plasmid and a helper plasmid encoding AAV2 Rep/Cap were cotransfected into HEK293T cells by calcium phosphate precipitation. Crude viral lysate was purified by $\mathrm{CsCl}$ gradient centrifugation, dialyzed, and concentrated with an Amicon filter. The viral genome titer was determined by qRT-PCR.

Surgery. The rats were briefly handled by the experimenter (twice for $10 \mathrm{~min}$ ) before the surgery day. For head plate implantation, rats were anesthetized with isoflurane $(4.5 \%$ for induction and $2.0-2.5 \%$ for maintenance; Pfizer) using an inhalation anesthesia apparatus (Univentor 400 anesthesia unit) and placed on a stereotaxic frame (SR-10R-HT; Narishige). In addition, lidocaine jelly (AstraZeneca) was administered around the surgical incisions for local anesthesia. During anesthesia, body temperature was maintained at $37^{\circ} \mathrm{C}$ using an animal warmer (BWT-100; Bio Research Center). The head plate (CFR-2; Narishige) was attached to the skull with small anchor screws and dental resin cement (Super-Bond C\&B, Sun Medical; Unifast II, GC Corporation). The reference and ground electrodes (Teflon-coated silver wires $125 \mu \mathrm{m}$ in diameter; A-M Systems) were implanted above the cerebellum. In some experiments ( $n=7$ rats), a twisted Teflon-coated silver wire electrode was implanted into the right upper forelimb (near the biceps brachii muscle) to measure EMG activity. For the optogenetic inhibition (GABA release) study (see Fig. 9), $1.5 \mu \mathrm{l}$ of AAV-CAGGS-DIO-ChRWR-Venus vector solution $\left(1-2 \times 10^{13} \mathrm{vg} / \mathrm{ml}\right)$ was injected into M1 and PPC of the VGAT-Cre rats (1300 $\mu \mathrm{m}$ in depth, see below for the coordinates; Legato100, Kd Scientific). Analgesics and antibiotics were applied postoperatively as required (meloxicam, $1 \mathrm{mg} / \mathrm{kg}$, s.c.; Boehringer Ingelheim; gentamicin ointment, $0.1 \%$ ad usum externum, MSD).

Water deprivation was started after full recovery from surgery $(6 \mathrm{~d}$ later). The rats had ad libitum access to water during weekends, but obtained water only by performing the task correctly during the rest of the week. When necessary, an agar block (containing $15 \mathrm{ml}$ of water) was given to the rats in their home cage to maintain them at $>85 \%$ of original body weight (Saiki et al., 2014; Soma et al., 2017).

Behavioral task. We used the right-left pedal task in our operant conditioning system (custom-made by O'Hara and Co., Ltd.; see Fig. $1 A$; also see Soma et al., 2017) to examine the behavioral and neural selectivity of contralateral or ipsilateral forelimb movement. In this task, the rats had to manipulate the right and left pedals with the corresponding forelimb in a head-fixed condition. They spontaneously started each trial by pushing both pedals down with the right and left forelimbs and holding them for a short period ("holding period," at least $1 \mathrm{~s}$; within a holding area, $0-30 \%$ in relative pedal position). After the holding period was completed, rats had to release either the right or left pedal depending on the context without any instruction cue to obtain $0.1 \%$ saccharin water $(10$ $\mu l)$ as a reward. The reward was dispensed from the tip of a spout by a 
micropump with a $300-700 \mathrm{~ms}$ delay (100 ms steps at random). This task consisted of two blocks of right-rewarded trials and left-rewarded trials. Each block lasted until the rat performed $>30$ correct (rewarded) trials and achieved correct performance of $80 \%$ in the most recent 10 trials. If the rats incorrectly released the other pedal (error trial), then they were given an error sound and were not rewarded $(3 \mathrm{kHz}, 300 \mathrm{~ms})$. If they did not complete the holding period (immature trial), then they did not receive feedback. The rats typically learned this operant task within 2 weeks ( $2-3 \mathrm{~h}$ a day).

Once the rats completed task learning, they underwent a second surgery under isoflurane anesthesia for later recording/stimulation experiments. We made tiny holes (1.0-1.5 $\mathrm{mm}$ in diameter) in the skull and dura mater above the M1 $(1.0 \mathrm{~mm}$ anterior, $\pm 2.5 \mathrm{~mm}$ lateral from bregma; Soma et al., 2017; Saiki et al., 2018), M2 (3.5 mm anterior, \pm 2.4 $\mathrm{mm}$ lateral; Soma et al., 2017; Saiki et al., 2018), and PPC $(3.8 \mathrm{~mm}$ posterior, $\pm 2.4 \mathrm{~mm}$ lateral; Erlich et al., 2015; Hanks et al., 2015; Yoshida et al., 2018). The coordinates of M1 and M2 were determined by intracortical microstimulation (ICMS; $50-100 \mu \mathrm{A}, 50$ pulses at $100 \mathrm{~Hz}$ ) to evoke reliable movements of the contralateral forelimb in our previous studies (Saiki et al., 2014, 2018; Kimura et al., 2017; Soma et al., 2017). The coordinates of the PPC were determined by histological observations using retrograde tracers in our preliminary experiments (see Fig. $2 B, C$ ). All holes were immediately covered with silicon sealant (DentSilicone-V; Shofu) until the recording/stimulation experiments.

In vivo electrophysiological recording. We performed extracellular multineuronal (multiple isolated single-unit) recordings from individual neurons while the rats were performing the behavioral task. Supported by agarose gel ( $2 \%$ agarose-HGT; Nacalai Tesque) on the brain, $32-$ channel silicon probes (Isomura32-A32 or Iso_3x_tet-A32; NeuroNexus Technologies; Saiki et al., 2018) were precisely inserted into the M1, M2, and PPC. Insertions were performed using fine micromanipulators (SM-15 or SMM-200B; Narishige) at least $1 \mathrm{~h}$ before the start of the recording experiment.

The wide-band signals were amplified and filtered (FA64I; Multi Channel Systems; final gain, 2000; band-pass filter, $0.5 \mathrm{~Hz}$ to $10 \mathrm{kHz}$ ) through a 32-channel head stage (MPA32I; Multi Channel Systems; gain, 10). These signals were digitized at $20 \mathrm{kHz}$ and recorded by three $32-$ channel hard-disc recorders (LX-120; TEAC), which simultaneously digitized the pedal positions tracked by angle encoders and the events of optogenetic stimulation. In some experiments, the EMG activity of the right forelimb was obtained by an amplifier with a head stage (EX4-400; Dagan; gain, 1000; band-pass filter, $0.3 \mathrm{~Hz}$ to $10 \mathrm{kHz}$ ), which was also digitized and recorded by the same hard-disk recorder.

Spike isolation. Raw signal data were processed offline to isolate spike events of individual neurons in each tetrode of the silicon probes. Briefly, spike candidates were detected and clustered by our semiautomatic spike-sorting software, EToS (Takekawa et al., 2010, 2012). Using the manual clustering software Klusters and the viewing software NeuroScope (Hazan et al., 2006), spike clusters were further combined, divided, and/or discarded manually to refine single-neuron clusters based on the presence of refractory periods $(<2 \mathrm{~ms})$ in their own autocorrelograms and from the absence of refractory periods in cross-correlograms with other clusters.

Spike analysis. In each neuron (spike cluster), basal spiking properties and functional activity in relation to behavioral task performance were analyzed using MATLAB (The MathWorks) as follows. The ongoing spike rate and spike duration (onset to peak) for individual spike clusters were defined in the same manner as in our previous studies (Isomura et al., 2009; Soma et al., 2017; Saiki et al., 2018). The spike clusters were classified as regular-spiking (RS, mostly putative excitatory) neurons and fast-spiking (FS, putative inhibitory) neurons based on the spike duration ( $\geq 0.6 \mathrm{~ms}$ for RS neurons, $<0.6 \mathrm{~ms}$ for FS neurons). As we discuss many groups of neurons (RS vs FS and M1 vs M2 vs PPC), we use abbreviated expressions to refer to them for simplicity (e.g., M1-RS for RS neurons in M1).

Next, we examined functional spike activity correlated with behavioral performance of forelimb movements. In this study, we focused on unilateral forelimb movements (contralateral and ipsilateral independently; i.e., excluding simultaneous bilateral movements) to evaluate the speci- ficity of functional spike activity to right-left forelimb control. Therefore, we analyzed spike trains in relation to unilateral forelimb movements during task performance ( $\geq 20$ trials with total $\geq 250$ spikes), which were aligned with the onset $(0 \mathrm{~s})$ of pedal release (following $\geq 1 \mathrm{~s}$ holding time). The release onset was defined as follows: the pedal release was first detected as the time when the pedal went outside of the holding area (30\% in relative pedal position) and, then, the onset was defined as the time when the pedal position exceeded $5 \%$ in relative pedal position before the approximate pedal release. Task-related activity was defined by the task relevance index using the Kolmogorov-Smirnov (KS) test, as described previously (also see Fig. $2 \mathrm{~A}$ in Soma et al., 2017; Kimura et al., 2017; Saiki et al., 2018); we defined a task-related neuron as a neuron with a task relevance index $(P)$ smaller than the criterion $\left(p=10^{-6}\right)$ in contralateral or ipsilateral pedal release trials. Its preference (contralateral or ipsilateral) was defined as the side with the smaller task relevance index. The task-related neurons were further classified into hold-type and go-type (sum of pre-go-type and post-go-type) according to the peak time position of spike increase and the dependence on pedal holding time in the perievent time histogram (PETH; $20 \mathrm{~ms}$ bins) on the preferred side (see Fig. 4A-D; Soma et al., 2017). Hold-type neurons have a sustained spike increase before the release onset $(0 \mathrm{~s})$ depending on the holding time. Go-type neurons have a phasic spike increase independent of the holding time; they are classified as the pre-go-type if the peak precedes the release onset $(0 \mathrm{~s})$ and otherwise as the post-go-type.

To determine the limb preference of a go-type neuron, we compared the peak amplitude between contralateral and ipsilateral release trials. The peak amplitude was calculated by averaging the spike rate in the peak period (the center of peak bin $\pm 150 \mathrm{~ms}$ ), in which the peak bin was determined in the PETH of preferred release trials (contralateral or ipsilateral). The same peak period was also used for calculating the peak amplitude in nonpreferred release trials. For a hold-type neuron, we compared the mean spike rate during the holding period $(-1000$ to 0 $\mathrm{ms}$ ) between contralateral and ipsilateral release trials. Moreover, we evaluated the limb preference (laterality) of go-type neurons using the laterality index (ranging from -1 to +1 ; Soma et al., 2017) based on normalized peak activities as follows:

$$
\text { Laterality index }=\left\{\begin{array}{cl}
(c-i) /(c+i), & \text { if } c>0 \text { and } i>0 \\
+1, & \text { if } c>0 \text { and } i<0 \\
-1, & \text { if } c<0 \text { and } i>0
\end{array}\right.
$$

where $c$ and $i$ are the activities associated with contralateral and ipsilateral movements, respectively. These parameters were obtained from the following equation:

$$
c, i=S R_{\text {peak }} / S R_{\text {baseline }}-1
$$

where $S R_{\text {peak }}$ is the mean spike rate in the peak period (the center of peak bin $\pm 150 \mathrm{~ms}$ ), and $S R_{\text {baseline }}$ is the mean spike rate in the baseline period ( -1000 to $-700 \mathrm{~ms}$ relative to the pedal release onset). Therefore, laterality index values of -1 and +1 indicate ipsilateral- and contralateralpreferring neuronal activity, respectively. The laterality index was applied to EMG activity, pedal trace, and video image analyses.

To characterize the limb preference of a go-type neuron without the effect of trial-to-trial variance, we performed a receiver operating characteristic (ROC) analysis to compare the distribution of spike rates (the center of peak bin $\pm 150 \mathrm{~ms}$ ) across trials between contralateral and ipsilateral release trials. An area under the ROC curve (AUC) $>0.5$ indicates a higher spike rate of contralateral release trials relative to that of ipsilateral, whereas an AUC $<0.5$ indicates a lower spike rate of contralateral release trials relative to that of ipsilateral.

The effects of trial block changes on spike activity were examined in the fraction of neurons showing a stronger preference for one side (laterality index $>0.5$ and $<-0.5$ for contralateral and ipsilateral, respectively). We averaged the peak activity separately at the first to tenth correct trial from the start of the preferred trial block and normalized them by the averaged peak activity of all correct trials.

EMG data analysis. The EMG signal was rectified and averaged to calculate the laterality index and onset latency (Nakayama et al., 2015; Soma et al., 2017). The laterality index was calculated between 0 and 
$+500 \mathrm{~ms}$ from the onset of pedal release. The onset latency was defined as the first time when 10 consecutive bins of EMG power ( $1 \mathrm{~ms}$ bin width) were above a $5 \mathrm{SD}$ threshold from the mean value in baseline period ( -1000 to $-700 \mathrm{~ms}$ from the pedal release onset).

Slice preparation for ex vivo optogenetics. Acute brain slices were prepared from VGAT-Cre rats 5-6 weeks after virus injection for whole-cell patch-clamp recordings according to procedures described previously (Sugimura et al., 2016). Briefly, the rats were transcardially perfused with ice-cold cutting solution under isoflurane anesthesia $\left(5 \%\right.$ in $100 \% \mathrm{O}_{2}$ ) and decapitated. The brain was then removed and forebrain blocks containing the M1 and PPC were dissected out in ice-cold cutting solution composed of the following (in mM ): $2.5 \mathrm{KCl}, 0.5 \mathrm{CaCl}_{2}, 10 \mathrm{MgSO}_{4}, 1.25$ $\mathrm{NaH}_{2} \mathrm{PO}_{4}, 2$ thiourea, 3 sodium pyruvate, $93 \mathrm{~N}$-methyl-D-glucamine, 20 HEPES, $12 \mathrm{~N}$-acetyl-L-cysteine, $25 \mathrm{D}$-glucose, $5 \mathrm{~L}$-ascorbic acid, and 30 $\mathrm{NaHCO}_{3}$ equilibrated with $95 \% \mathrm{O}_{2}+5 \% \mathrm{CO}_{2}$, pH 7.1-7.5; osmolality, $290 \mathrm{mOsmol} / \mathrm{kg} \mathrm{H}_{2} \mathrm{O}$. The brain blocks were embedded in agarose (1.6\%; Sigma-Aldrich) and brain slices of $300 \mu \mathrm{m}$ thickness were prepared with a vibrating blade slicer (Neo linear slicer NLS-MT; Dosaka). The slices were incubated in the cutting solution at $34^{\circ} \mathrm{C}$ for $15 \mathrm{~min}$ and then transferred into artificial CSF (ACSF) containing the following (in mM): $125 \mathrm{NaCl}, 3 \mathrm{KCl}, 2 \mathrm{CaCl}_{2}, 2 \mathrm{MgCl}_{2}, 1.25 \mathrm{NaH}_{2} \mathrm{PO}_{4}, 10$ D-glucose, $0.4 \mathrm{~L}$-ascorbic acid, and $25 \mathrm{NaHCO}_{3}$, pH 7.4, bubbled with $95 \% \mathrm{O}_{2}+5 \%$ $\mathrm{CO}_{2}$; osmolality, $\sim 310 \mathrm{mOsmol} / \mathrm{kg} \mathrm{H}_{2} \mathrm{O}$ at room temperature (20$25^{\circ} \mathrm{C}$ ) until recording. Each slice was submerged in a recording chamber and superfused with the ACSF continuously at a rate of $2 \mathrm{ml} / \mathrm{min}$ at room temperature. For the recording of light evoked IPSCs, the ACSF also contained $3 \mathrm{~mm}$ kynurenic acid (Sigma-Aldrich). Bicuculline methiodide (10 $\mu \mathrm{M}$ in ACSF; Sigma-Aldrich) was bath-applied to block $\mathrm{GABA}_{\mathrm{A}}$ receptor response.

Patch-clamp recordings from M1 and PPC neurons. M1 and PPC neurons were identified visually under an upright microscope (BX-51WI; Olympus) with oblique illumination. Epifluorescence images were captured using a CCD (IR-1000; DAGE-MTI) or a CMOS camera (AdvanCam-HD1080; AdvanVision). Patch-clamp electrodes (3-8 M $\Omega$ ) made from borosilicate glass pipettes (1B120F-4; World Precision Instruments) contained an internal solution consisting of the following (in mM): 120 potassium gluconate, $6 \mathrm{NaCl}, 1 \mathrm{CaCl}_{2}, 2 \mathrm{MgCl}_{2}, 2 \mathrm{ATP} \mathrm{Mg}, 0.5$ GTP Na, 12 phosphocreatine $\mathrm{Na}_{2}, 5$ EGTA, and 10 HEPES hemisodium, along with $0.00125-0.0025 \%$ Alexa Fluor 594 (A10438; Life Technologies) ( $\mathrm{pH} 7.3$; osmolality, $290-300 \mathrm{mOsmol} / \mathrm{kg} \mathrm{H}_{2} \mathrm{O}$ ). Whole-cell currents were recorded using an IPA amplifier (Sutter Instruments), filtered at $2 \mathrm{kHz}$ (voltage-clamp recordings) or $5 \mathrm{kHz}$ (current-clamp recordings), and digitized at $10 \mathrm{kHz}$ with 16-bit resolution using a PowerLab interface. The cells expressing ChRWR-Venus (Venus ${ }^{+}$cells) were identified with epifluorescence video micrograph. We recorded from the Venus $^{+}$cells and Venus ${ }^{-}$cells near Venus ${ }^{+}$terminals.

Light stimulation to slice preparations. ChRWR was activated using a high-power light-emitting diode (LED) illumination system $(470 \mathrm{~nm}$, M470F3; Thorlabs; or $465 \mathrm{~nm}, \mathrm{LEX}-2 \mathrm{~B}$; Brainvision) controlled by Master-8 (A.M.P.I.). Illumination was delivered to the whole field through a $40 \times, 0.8$ numerical aperture (NA) objective (LumPlan FL N; Olympus). We used $5 \mathrm{~ms}$ duration and $\sim 200 \mathrm{~mW} / \mathrm{mm}^{2}$ intensity. Two or 10 light stimuli were delivered every $10 \mathrm{~s}$.

Optogenetic activation and optogenetic inhibition via GABA release. We optogenetically stimulated ChR2-expressing neurons in the Thy1-ChR2 rats (see standard optogenetic activation in Figs. 7 and 8, weak optogenetic activation in Fig. 10) and ChRWR-expressing GABAergic neurons in the VGAT-Cre rats (see optogenetic inhibition via GABA release in Fig. 9). Optical fibers (FT400EMT, FC, Thorlabs; core diameter $400 \mu \mathrm{m}$, NA 0.39 for standard optogenetic activation; and $1000 \mu \mathrm{m}$, NA 0.39 for optogenetic inhibition via GABA release and weak optogenetic activation) were placed on the surface of M1, M2, and/or PPC using micromanipulators (SM-25A; Narishige). A pulse of blue LED light (460 nm, $10-35 \mathrm{~mW}$ ) was applied unilaterally through the optical fiber using an ultra-high-power LED light source (UHP-Mic-LED-460, FC; Prizmatix) triggered by a stimulator (SEN-8203; Nihon Kohden). The intensity was adjusted by changing the pulse duration and frequency depending on the purpose of the experiments.
To evaluate the effect of optogenetic activation or optogenetic inhibition via GABA release on behavioral task performance, 10 test $(\mathrm{ON})$ and 10 control (OFF) trials were set randomly in the middle of each block (e.g., black dashed box in Fig. 9E) to avoid influence from block changes. To prevent the rats from using the light as a visual cue, the optical fibers on the brain surface were covered with paraffin wax containing pontamine sky blue (Direct Blue 1, Tokyo Kasei; Soma et al., 2012).

Evaluation of motor response evoked by optogenetic activation. To evaluate forelimb response evoked by optogenetic activation, we obtained the laterality index (0-500 ms after the stimulation) and the release latency of pedal traces that were aligned with the onset of optogenetic activation on the preferred side (contralateral for motor cortices; ipsilateral for PPC). The latency was the time until the pedal went outside of the holding area (30\% in relative pedal position) after optogenetic activation.

In video analysis, front-view images were acquired using an infrared video camera (w480 $\times$ h854 pixels; MK-323, YK Musen) at a frame rate of $30 \mathrm{~Hz}$ (GV-MDVD3, I-O DATA device). To detect forelimb deviation, we set the regions of interest $(95 \times 225$ pixels) onto equivalent portions of the two forelimbs and calculated a pixel-wise absolute difference (PWAD) between 0 and $300 \mathrm{~ms}$ before and after optogenetic activation (Powell et al., 2015; Goard et al., 2016). The PWAD was obtained from the following equation:

$$
P W A D_{i}=\operatorname{Frame}_{i}(x, y)-\text { Frame }_{i-1}(x, y)
$$

where Frame $_{\mathrm{i}}$ is the video frame at time point $i$. We averaged $P W A D_{\mathrm{i}}$ within the frame and calculated the time course of forelimb movements over the consecutive 18 frames ( -300 to $+300 \mathrm{~ms}$ from the onset of optogenetic activation) and the laterality index in PWAD.

Evaluation of effects of optogenetic activation or inhibition on task performance. To assess the effects of optogenetic cortical manipulation on task performance, we calculated the net effect of optogenetic inhibition via GABA release or weak optogenetic activation using the following equation:

$$
\text { Net effect }=\text { effect } t_{c}-\text { effect }
$$

where effect $t_{c}$ and effect $t_{\mathrm{i}}$ show the effect of weak optogenetic activation or inhibition on task performance in contralateral- and ipsilateralrewarded blocks, respectively. These parameters were obtained from the following equation:

$$
\text { effect }=\frac{C R_{o n}-C R_{\text {off }}}{C R_{o n}+C R_{o f f}}
$$

where $C R_{\text {on }}$ and $C R_{\text {off }}$ are the correct rate (\%) in task performance for test trials (with optogenetic activation or inhibition) and control trials (without optogenetic activation or inhibition), respectively, in each block type. Positive and negative values in the net effect of optogenetic activation or inhibition denote a shift of pedal release preference to contralateral and ipsilateral, respectively.

Histological observations. After the recording/stimulation experiments, animals were deeply anesthetized with urethane $(2-3 \mathrm{~g} / \mathrm{kg}$, i.p.; Nacalai Tesque) and transcardially perfused with cold saline followed by $4 \%$ formaldehyde in $0.1 \mathrm{~m}$ phosphate buffer. Whole brains were postfixed and sliced coronally into $50 \mu \mathrm{m}$ serial sections using a microslicer (VT1000S; Leica). Electrode tracks labeled with 1, $1^{\prime}$-dioctadecyl-3,3,3', $3^{\prime}$ tetramethylindocarbocyanine perchlorate (DiI, Thermo Fisher Scientific) were investigated in the motor cortices and PPC under a fluorescence microscope (BX51N; Olympus). The Cre-dependent expression of tdTomato in GABAergic interneurons was assessed in the brains of double-transgenic rats obtained from the crossing of a tdTomato reporter rat and a VGAT-Cre rat (see Fig. 9A). GABA immunostaining was performed as described previously (Igarashi et al., 2018). Briefly, GABA-immunoreactive neurons were visualized with an anti-GABA mouse antibody (1:300, MAB316; Millipore Bioscience Research Reagents) and an Alexa Fluor 568-conjugated secondary antibody (1:300; Invitrogen). The sections were coverslipped with Vectashield Antifade Mounting Medium with 4',6-diamidino-2-phenylindole (DAPI; Vector Laboratories) and observed by laser scanning microscope (FV1200; 
A

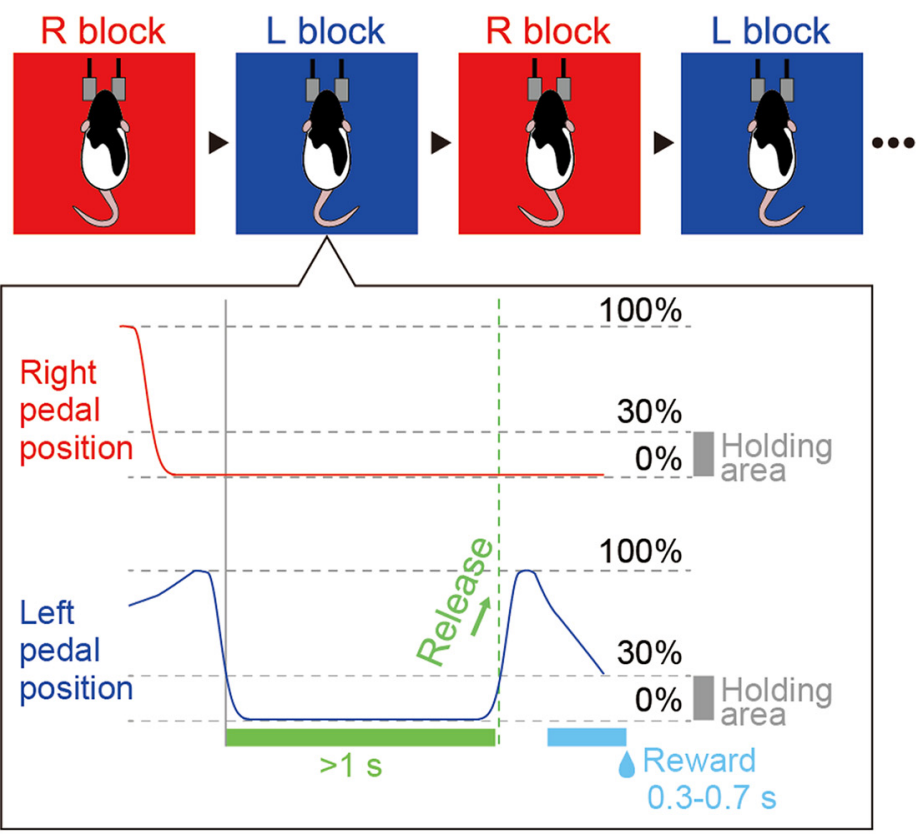

B
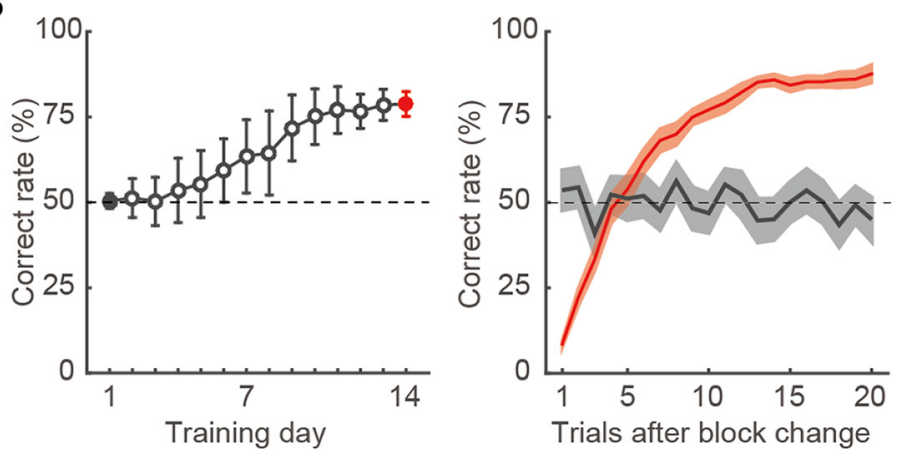

C

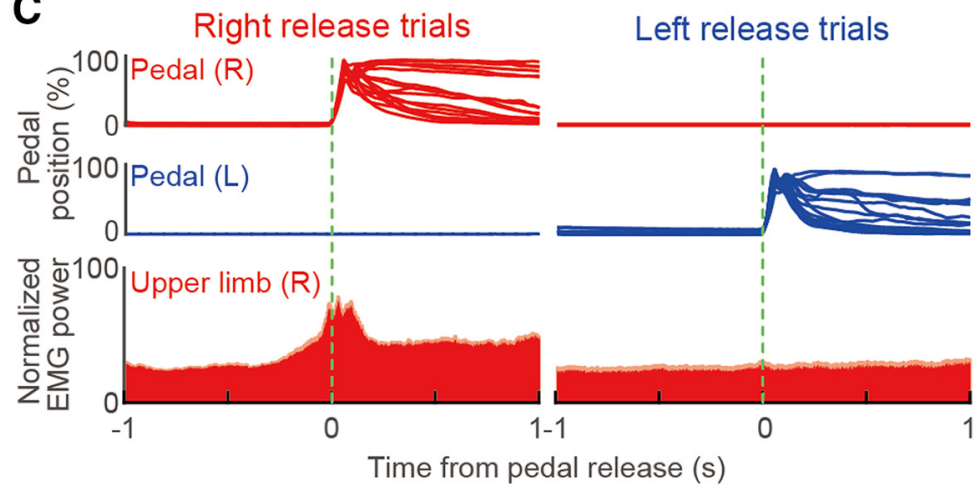

Figure 1. Behavioral task performance. $A$, Schematic diagram of the right-left pedal task. Head-fixed rats pushed down both pedals for a short period $(\geq 1 \mathrm{~s}$ ) to initiate each trial and then released either of the pedals (e.g., left release) voluntarily (without cue) to acquire a reward. This task consisted of right-rewarded (R) and left-rewarded (L) blocks, which were alternated after meeting the criteria (see Materials and Methods). $\boldsymbol{B}$, Learning curve over 14 training days (left). Shown is the averaged correct rate in the first to $20^{\text {th }}$ trials after the change of blocks (right). Gray and red colors represent the first and last training days, respectively. C, Right and left pedal trajectories (overlaid) and EMG activity in right forelimb (normalized EMG power, mean + SEM) in relation to the onset of pedal release (green dashed line) for right and left release trials.

Olympus). The fluorescent labeling of ChRWR-Venus in the VGAT-Cre rats was assessed under the microscope after fluorescent Nissl staining (NeuroTrace; Thermo Fisher Scientific; see Fig. 9C).

Experimental design and statistical analyses. We obtained electrophysiological data from 48 sessions in 17 Thy1-ChR2 rats to examine forelimb-movement representations of the M1, M2, and PPC neurons.
We combined the new electrophysiological data with data from our previous study (M1, $n=12$ rats; $\mathrm{M} 2, n=13$ rats; Soma et al., 2017). In total, we included data from $838 \mathrm{M} 1$ neurons, 1583 M2 neurons, and 915 neurons during task performance $(\mathrm{M} 1,57 \pm 28$ cells; $\mathrm{M} 2$, $70 \pm 27$ cells; PPC, $69 \pm 31$ cells per session). These neurons were divided into RS and FS subclasses by spike duration and were further classified into go- and hold-type neurons if they were related with task events functionally (see Figs. 1, 2, 3, 4, 5, and 6). We also used 16 Thy1-ChR2 rats to observe optogenetically evoked forelimb movements (see Figs. 7 and 8 ) and four VGAT-Cre rats (see Fig. 9E-H; 12 sessions) and four Thy1-ChR2 rats (see Fig. 10; 12 sessions) to evaluate the optogenetic effect on their task performance. Data in the text and figures are expressed as means \pm SD (unless otherwise noted) and sample number $(n)$. Sample sizes (number of animals, sessions, and neurons) were estimated according to previous studies (Goard et al., 2016; Soma et al., 2017) and confirmed to be adequate by power analyses (power $=0.9 ; \alpha$ error $=0.05$ ). We used the following statistical methods: one-sample signed-rank test, Mann-Whitney test, Wilcoxon signed-rank test, $\chi^{2}$ test, KS test, Kruskal-Wallis test, Friedman test, and twoway ANOVA. All tests were two sided unless otherwise stated. These statistical tests were conducted by using MATLAB's Statistics and Machine Learning Toolbox (The MathWorks). Differences were considered statistically significant when $p<0.05$ (see Results for details). Blinding and randomization were not performed.

\section{Results}

\section{Behavioral performance}

To examine preference for contralateral or ipsilateral forelimb movements (limb preference) in spike activity of each neuron, we used the right-left pedal task, in which rats manipulated two pedals separately with their right or left forelimb in a steady posture under head fixation (Fig. $1 A$; see also Soma et al., 2017). In this task, the rats started each trial by pushing both pedals down. After a brief holding period, they had to spontaneously (with no cue) release either the right or left pedal by moving the corresponding forelimb for reward acquisition. In every trial, they were rewarded for their appropriate release of the right or left pedal in a block manner (right- and left-rewarded blocks; see Materials and Methods for details). The rats successfully learned to perform the right-left pedal task in 2 weeks (Fig. $1 B$, left). After task training, the rats properly changed their forelimb movement from one side to the other in four to six trials from the start of block (Fig. 1B, right; 14th training day; see also Fig. 9E).

In this study, EMG activity was measured in the right upper forelimb during task performance, which confirmed a specific 
A

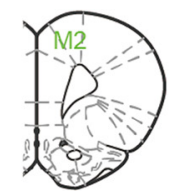

Bregma $3.24 \mathrm{~mm}$
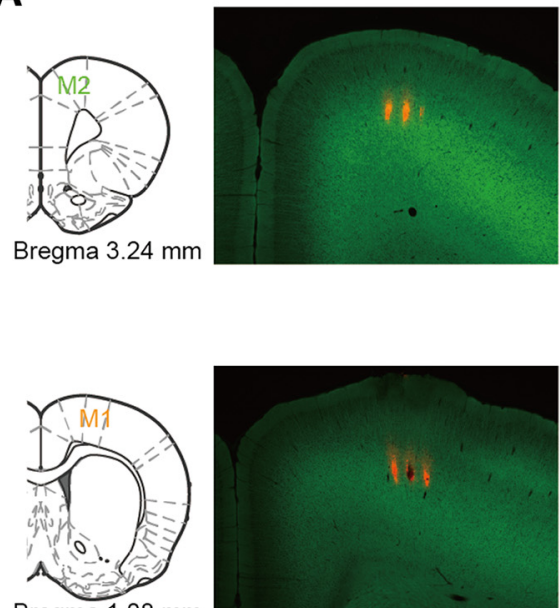

Bregma $1.08 \mathrm{~mm}$
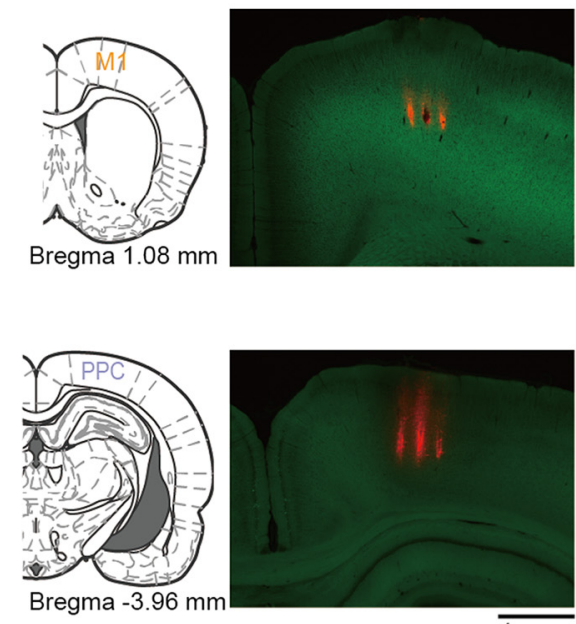

$\overline{\mathrm{mm}}$
B

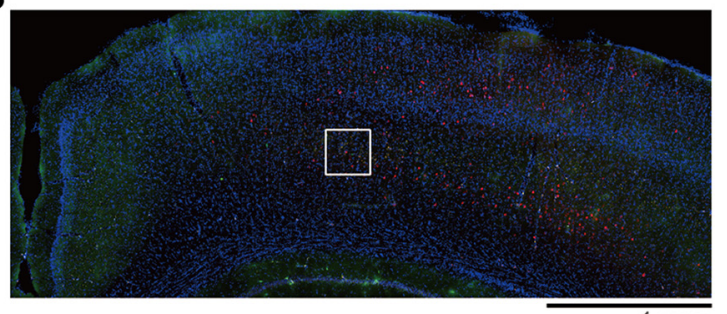

$1 \mathrm{~mm}$
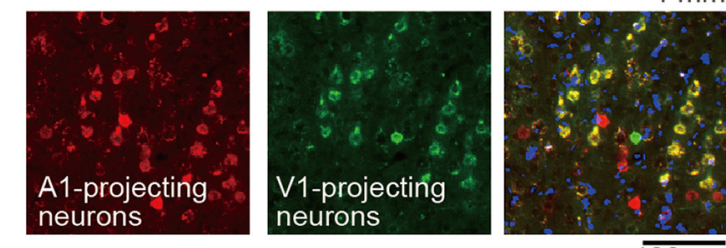

$\overline{100 \mu \mathrm{m}}$

C
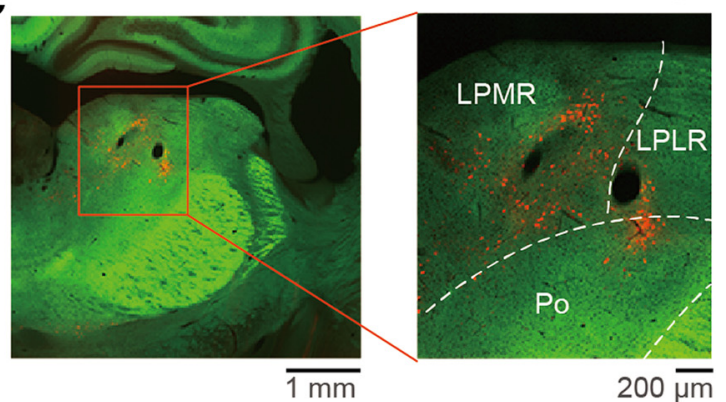

Figure 2. Histological confirmation of PPC coordinate and recording sites. $A$, Recording sites in the M2 (top), M1(middle), and PPC (bottom). Probe shank tracks were visualized by fluorescent Dil (red). B, PPC neurons sending axons to both visual and auditory cortices. The retrograde tracer cholera toxin subunit B (CTB)-conjugated Alexa Fluor 555 (red) and Alexa Fluor 488 (green) were injected into the auditory and visual cortices, respectively. Neurons were counterstained with DAPI (blue). C, Thalamic lateral posterior nucleus neurons sending axons to the PPC. The CTB tracer (red) was injected into the coordinates of the PPC determined in $\boldsymbol{B}$. LPLR, Lateral posterior nucleus, laterorostral part; LPMR, lateral posterior nucleus, mediorostral part; Po, posterior thalamic nuclear group.

increase in muscular activity of the right forelimb in association with the right pedal release (Fig. $1 C ; n=18$ sessions in 7 rats: right release trials, $36.8 \pm 13.6 \%$; left release trials, $1.4 \pm 3.7 \%)$. The laterality index of EMG activity was -0.85 (meaning specific to the right; see Materials and Methods) and the increase in EMG activity started $226 \mathrm{~ms}$ before the onset of pedal release. These observations clearly indicate that the rats executed a pedal release using only the forelimb on the same side, which was consistent with the pedal traces.

\section{Functional activity during forelimb movements in motor cortex and PPC neurons}

Multineuronal spike activity (i.e., multiple isolated single units) was recorded through silicon probes in the motor cortices (M1 and M2) and PPC of 17 rats performing the right-left pedal task. The coordinates of the recording sites were determined according to previous studies (Fig. 2A; PPC, Hanks et al., 2015; Erlich et al., 2015; M1 and M2, Soma et al., 2017; Saiki et al., 2018; Yoshida et al., 2018). In addition, the coordinates of the PPC were confirmed histologically based on the distribution of axonal projections (Fig. $2 B, C$ ). Briefly, the PPC should send axonal outputs to both visual and auditory cortices (Corwin and Reep, 1998; Whitlock et al., 2008; Wilber et al., 2014b) and receive axonal inputs from the lateral posterior nucleus of the thalamus (Chandler et al., 1992; Olsen and Witter, 2016).

We isolated a total of $838 \mathrm{M} 1$ neurons, $1583 \mathrm{M} 2$ neurons, and 915 PPC neurons during task performance. These neurons were further classified as RS or FS subtypes based on clear bimodality of spike duration (data not shown; see also Soma et al., 2017). The ongoing spike rates of FS subtypes were significantly higher than those of RS subtypes in the M1, M2, and PPC (M1-RS: $2.8 \pm 3.5$ Hz; M1-FS: $8.6 \pm 10.6 \mathrm{~Hz}$, Mann-Whitney test, $z=-9.34, p<$ $1.0 \times 10^{-20}$; M2-RS: $2.2 \pm 2.6 \mathrm{~Hz}$; M2-FS: $7.1 \pm 7.4 \mathrm{~Hz}, z=$ $-9.80, p<1.1 \times 10^{-22}$; PPC-RS: $2.3 \pm 3.3 \mathrm{~Hz}$; PPC-FS: $9.1 \pm$ $\left.9.2 \mathrm{~Hz}, z=-11.8, p<5.3 \times 10^{-32}\right)$, which is consistent with previous reports (Isomura et al., 2009; Saiki et al., 2014, 2018; Kimura et al., 2017; Soma et al., 2017).

Figure 3 shows examples of left M1 and PPC neuronal activity. The M1 neuron showed a phasic increase in spike rate that was directly associated with the movement of the contralateral, but not ipsilateral, forelimb (namely, contralateral preference; Fig. 3, left). Conversely, the PPC neuron exhibited phasic spike activity in association with both contralateral and ipsilateral forelimb movements (bilateral preference; Fig. 3, middle); however, the activity was stronger with ipsilateral movement. The third example is another PPC neuron showing sustained activity during the holding period. The activity rapidly deceased during forelimb movements (Fig. 3, right; see also Fig. 4A). To compare functional (task-related) spike activity for preferred forelimb movements among the M1, M2, and PPC, we classified the recorded RS and FS neurons into three functional types: the hold-type, prego-type, and post-go-type (Fig. 4A-D), according to our definition established previously (Soma et al., 2017; see also Materials and Methods). Hold-type denotes a task-related neuron with sustained activity during the holding period (Fig. 4A). Pre-go-type denotes a neuron with a peak of phasic activity just before pedal release independent of holding time (Fig. 4B). Post-go-type denotes a neuron with a peak following pedal release. We observed 
Left M1

Right release trials Left release trials
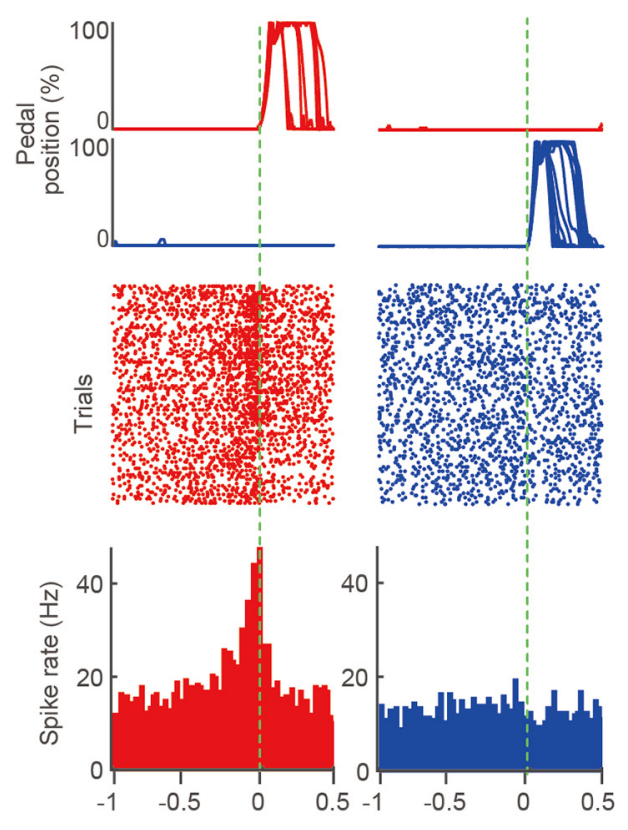

Left PPC

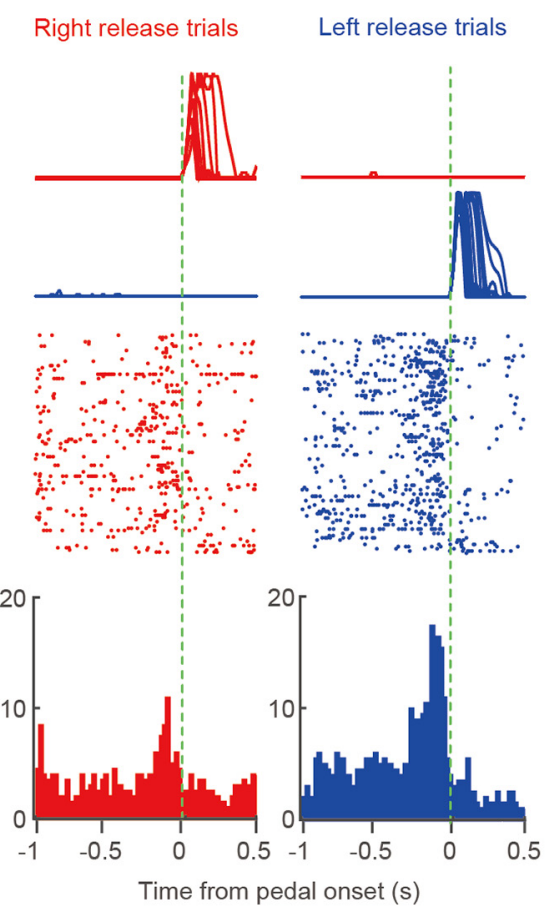

Left PPC

Right release trials Left release trials

Figure 3. Extracellular recordings during behavioral task performance. Examples of left M1 and PPC neurons showing functional (task-related) spike activity. Top, middle, and bottom show pedal trajectories, spike raster plots, and PETHs, respectively.

a bimodal distribution in the dependency of spike activity on the holding time, clearly dividing the hold-type and pre-go-type neurons (Fig. $4 C$; threshold at -0.5 in slope). Because the peak times of pre-go-type and post-go-type neurons were distributed continuously, the neurons were grouped together as go-type neurons for further analyses (Fig. 4D; all task-related neurons, M1-RS: $n=290$; M1-FS: $n=95$; M2-RS: $n=564$, M2-FS: $n=91$; PPC-RS: $n=277$; PPC-FS: $n=115$ ).

In accordance with our previous study on motor cortex neurons (Soma et al., 2017), the hold-type proportion in RS neurons was significantly larger than that in FS neurons in the M1, M2, and PPC (Fig. $4 D$; M1: $2 \times 2 \chi^{2}$ test, $\chi^{2}=13.1, p<2.9 \times 10^{-4}$; M2: $\chi^{2}=12.9, p<3.4 \times 10^{-4}$; PPC: $\left.\chi^{2}=4.7, p<3.0 \times 10^{-3}\right)$. We also found the peak time of go-type neurons in the PPC was significantly faster relative to M2 (Fig. 4E; RS: Kruskal-Wallis test, $H=14.45, p<7.3 \times 10^{-4}$; post hoc Scheffe's test, M2 vs PPC, $p<1.1 \times 10^{-3}$; FS: $\mathrm{H}=7.33, p<2.6 \times 10^{-2}$; M2 vs PPC, $\left.p<2.7 \times 10^{-2}\right)$.

\section{Preference for contralateral or ipsilateral forelimb movements in motor cortex and PPC neurons}

We compared functional spike activity between the contralateral and ipsilateral forelimb movements in all task-related neurons of M1, M2, and PPC (Fig. 5A). As described previously (Soma et al., 2017), M1 neurons typically showed preference for contralateral forelimb movements (as exemplified in Fig. 3, left) and the M2 neurons showed bilateral preference with a contralateral bias. PPC neurons, both the RS and FS subtypes, exhibited bilateral preference with bias not to the contralateral, but rather to ipsilateral forelimb movements (Fig. 3, middle). To quantify these opposing trends, we plotted the relation in peak activity of the gotype neurons between contralateral and ipsilateral forelimb movements for M1, M2, and PPC (Fig. 5B; see Materials and Methods). The peak activities of go-type M1-RS and M1-FS neu- rons were visibly larger with contralateral movements compared with ipsilateral movements, indicating their contralateral preference (M1-RS: contralateral, $6.7 \pm 6.6 \mathrm{~Hz}$, ipsilateral, $4.9 \pm 5.3$ $\mathrm{Hz}$, Wilcoxon signed-rank test, $z=6.93, p<4.1 \times 10^{-12}$; M1FS: contralateral, $16.6 \pm 17.1 \mathrm{~Hz}$, ipsilateral, $12.3 \pm 15.0 \mathrm{~Hz}, z=$ $5.50, p<3.8 \times 10^{-8}$ ). The go-type M2-RS and M2-FS neurons showed bilateral preference with contralateral bias (M2-RS: contralateral, $5.4 \pm 6.5 \mathrm{~Hz}$, ipsilateral, $4.7 \pm 5.2 \mathrm{~Hz}, z=4.77, p<$ $1.8 \times 10^{-6}$; M2-FS: contralateral, $14.4 \pm 14.4 \mathrm{~Hz}$, ipsilateral, $12.7 \pm 13.4 \mathrm{~Hz}, z=2.94, p<3.3 \times 10^{-3}$ ). In the go-type PPC neurons, both RS and FS subtypes displayed significantly larger peak activity for ipsilateral movements than for contralateral movements (PPC-RS: contralateral, $5.1 \pm 6.6 \mathrm{~Hz}$, ipsilateral, $5.6 \pm 7.3 \mathrm{~Hz}, z=-4.84, p<1.3 \times 10^{-6}$; PPC-FS: contralateral, $11.0 \pm 10.7 \mathrm{~Hz}$, ipsilateral, $12.7 \pm 12.4 \mathrm{~Hz}, z=-3.05, p<2.3 \times$ $\left.10^{-3}\right)$. Consistent with the difference in ongoing activity, the peak activity of go-type of FS neurons was higher than that of RS neurons in three cortical areas (M1, Mann-Whitney test between RS and FS, contralateral, $z=-6.01, p<1.89 \times 10^{-9}$, ipsilateral, $z=-5.87, p<4.48 \times 10^{-9} ; \mathrm{M} 2$, contralateral, $z=-7.20, p<$ $2.17 \times 10^{-12}$, ipsilateral, $z=-6.49, p<8.36 \times 10^{-11}$; PPC, contralateral, $z=-5.89, p<3.94 \times 10^{-9}$, ipsilateral, $z=-6.46$, $\left.p<1.06 \times 10^{-10}\right)$.

Hold-type neurons showed no bias in limb preference during sustained spike activity before movement in the M1, M2, and PPC (Fig. 5B; M1-RS: contralateral, $4.3 \pm 4.0 \mathrm{~Hz}$, ipsilateral, $3.8 \pm 3.1 \mathrm{~Hz}, z=1.91, p=0.06$; M1-FS: contralateral, $12.2 \pm$ $10.2 \mathrm{~Hz}$, ipsilateral, $12.3 \pm 9.1 \mathrm{~Hz}, z=5.29, p=0.90$; M2-RS: contralateral, $3.7 \pm 3.6 \mathrm{~Hz}$, ipsilateral, $3.8 \pm 3.8 \mathrm{~Hz}, z=-0.01$, $p=0.99 ;$ M2-FS: contralateral, $7.3 \pm 7.4 \mathrm{~Hz}$, ipsilateral, $7.4 \pm 7.7$ $\mathrm{Hz}, z=-0.66, p=0.54$; PPC-RS: contralateral, $4.3 \pm 6.2 \mathrm{~Hz}$, ipsilateral, $4.3 \pm 5.8 \mathrm{~Hz}, z=-0.55, p=0.58$; PPC-FS: contralateral, $10.1 \pm 9.8 \mathrm{~Hz}$, ipsilateral, $10.4 \pm 9.0 \mathrm{~Hz}, z=-1.23, p=0.22$ ). 
A

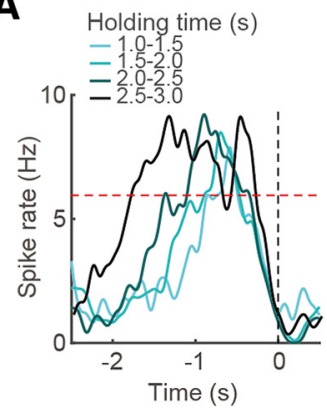

B

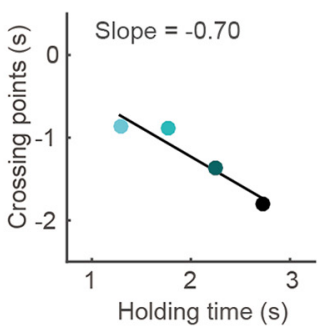

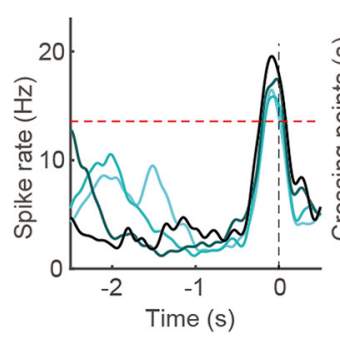

C

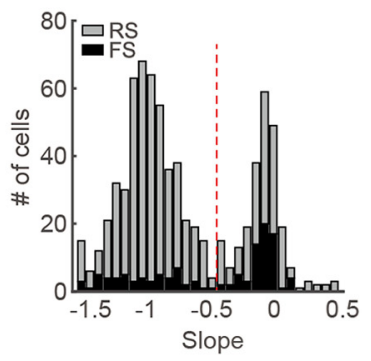

C

D

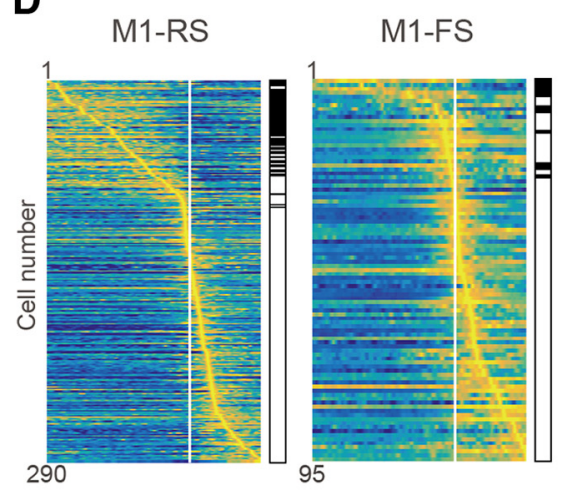

M2-RS

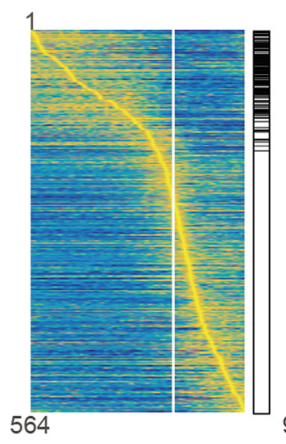

M2-FS

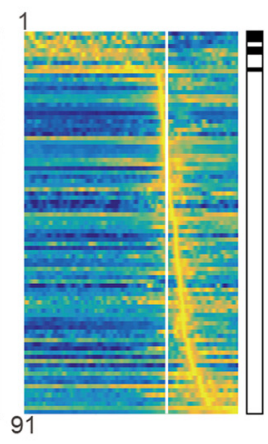

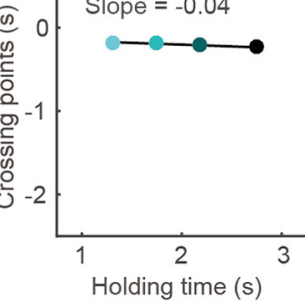

Slope $=-0.04$

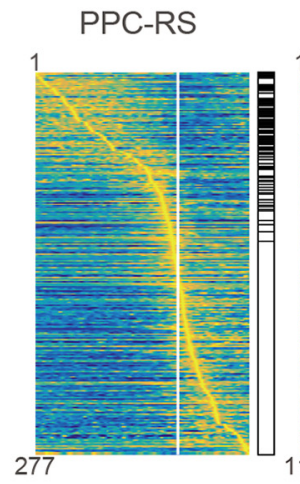

\section{PPC-FS}
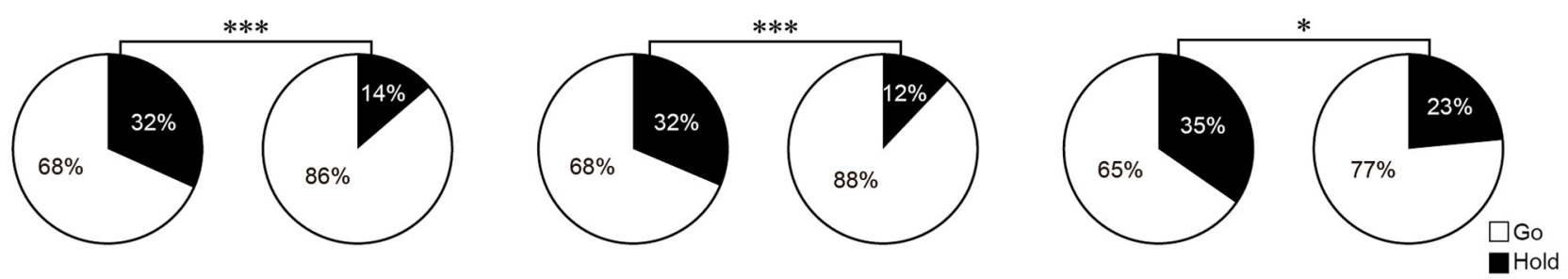

\section{E}
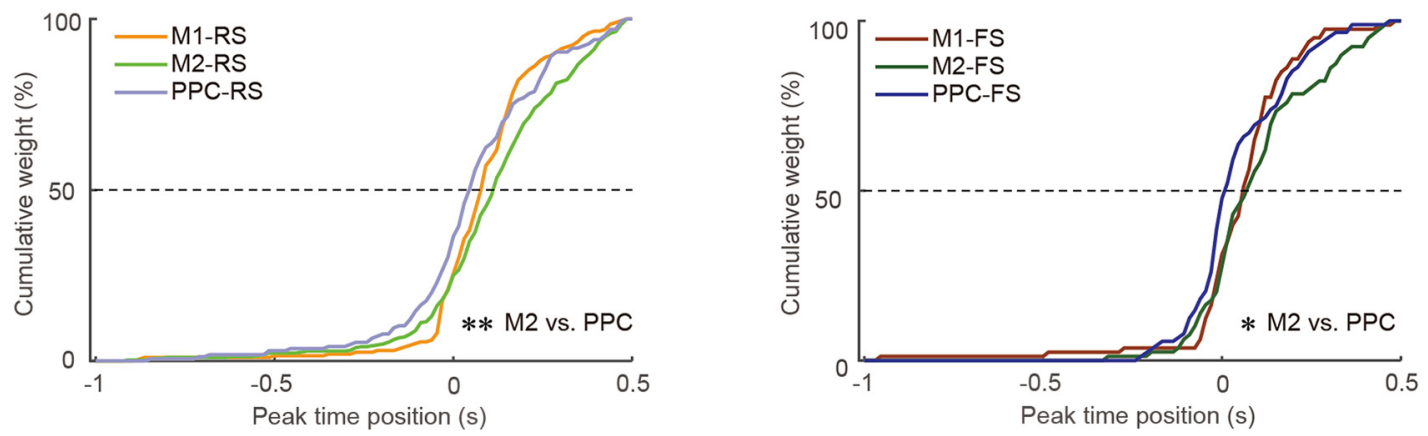

Figure 4. Functional (task-related) spike activity in M1, M2, and PPC neurons. $\boldsymbol{A}, \boldsymbol{B}$, Classification of hold-type $(\boldsymbol{A})$ and pre-go-type ( $\boldsymbol{B})$ activities according to the dependence of spike increase on behavioral holding time. Left, PETHs from a representative PPC neuron calculated from trials with varying ranges of holding time (light to dark blue). Right, Plot of the intersection with criterion (red dashed line, $75 \%$ of peak activity in all-averaged PETH) against the four ranges of holding time, in which we obtained the slope value from linear regression. The slope will be negative for hold-type $(\boldsymbol{A})$ and near zero for pre-go-type $(\boldsymbol{B})$ activities. C, Clear bimodal distribution of slope values in all task-related neurons with their peak time before the pedal release. Red dashed line indicates threshold for classification into hold- (toward negative) and pre-go-type (near zero). The third, post-go-type was defined as task-related neurons with their peak time after the pedal release. $\boldsymbol{D}$, Three types of functional activity in the RS and FS subtypes of M1, M2, and PPC neurons. Top, Normalized Gaussian-filtered PETHs ( $\sigma=12.5$ ms for spikes in 0.05 ms bins) aligned with the pedal release onset at $0 \mathrm{~s}$ (vertical line) for individual task-related neurons. Each row represents a single neuron, which were sorted by the order of peak time (early to late). The functional type is indicated on the right side (filled, hold; open, go). Bottom, Population ratios of functional types in the RS and FS neurons of M1, M2, and PPC. ${ }^{*} p<0.05,{ }^{* * *} p<0.001,2 \times 2 \chi^{2}$ test. E, Cumulative distributions of the peak time position of go-type activity for RS (left) and FS (right) subtypes of M1, M2, and PPC neurons. The pre- and post-go-type were grouped together as the go-type activity for analyses. The go-type activity emerged faster in PPC neurons than in M2 neurons. ${ }^{*} p<0.05,{ }^{* *} p<0.01$, post hoc Scheffe's test.

We evaluated the difference in limb preference of go-type neurons among the motor cortices and PPC using the laterality index of peak activity (Fig. 5C; see Materials and Methods). The laterality indices of $\mathrm{M} 1$ and $\mathrm{M} 2$ neurons were significantly biased toward a preference for contralateral movements (M1-RS: $0.39 \pm$
0.61 , one-sample signed-rank test, $z=7.36, p<1.8 \times 10^{-13}$; M1-FS: $0.28 \pm 0.56, z=4.05, p<5.1 \times 10^{-5}$; M2-RS: $0.16 \pm$ $0.67, z=4.60, p<4.3 \times 10^{-6}$ M2-FS: $0.21 \pm 0.47, z=3.88, p<$ $\left.1.1 \times 10^{-4}\right)$. In contrast, the laterality indices of both RS and FS subtypes of PPC neurons were biased toward ipsilateral move- 
A
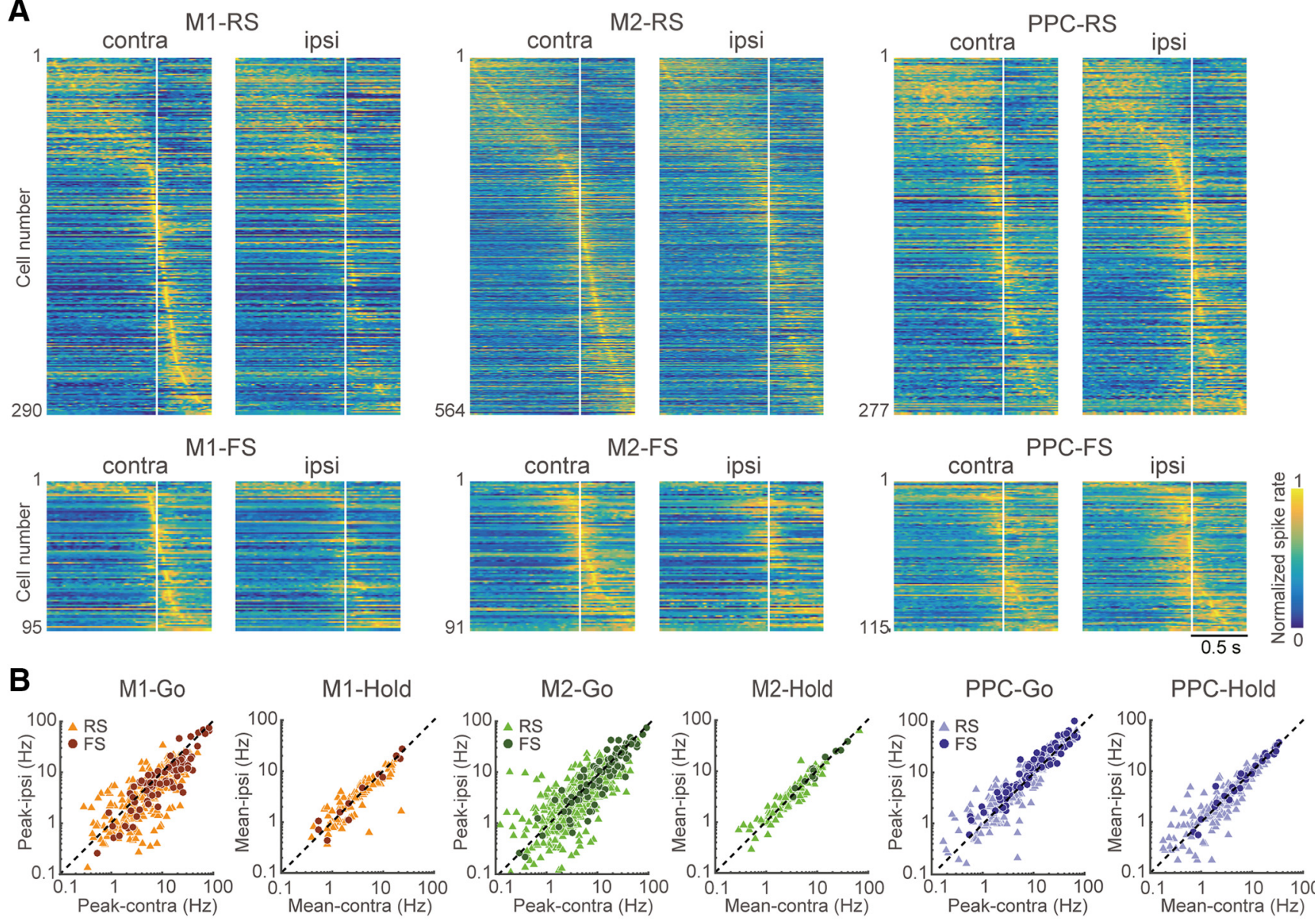

PPC-Go
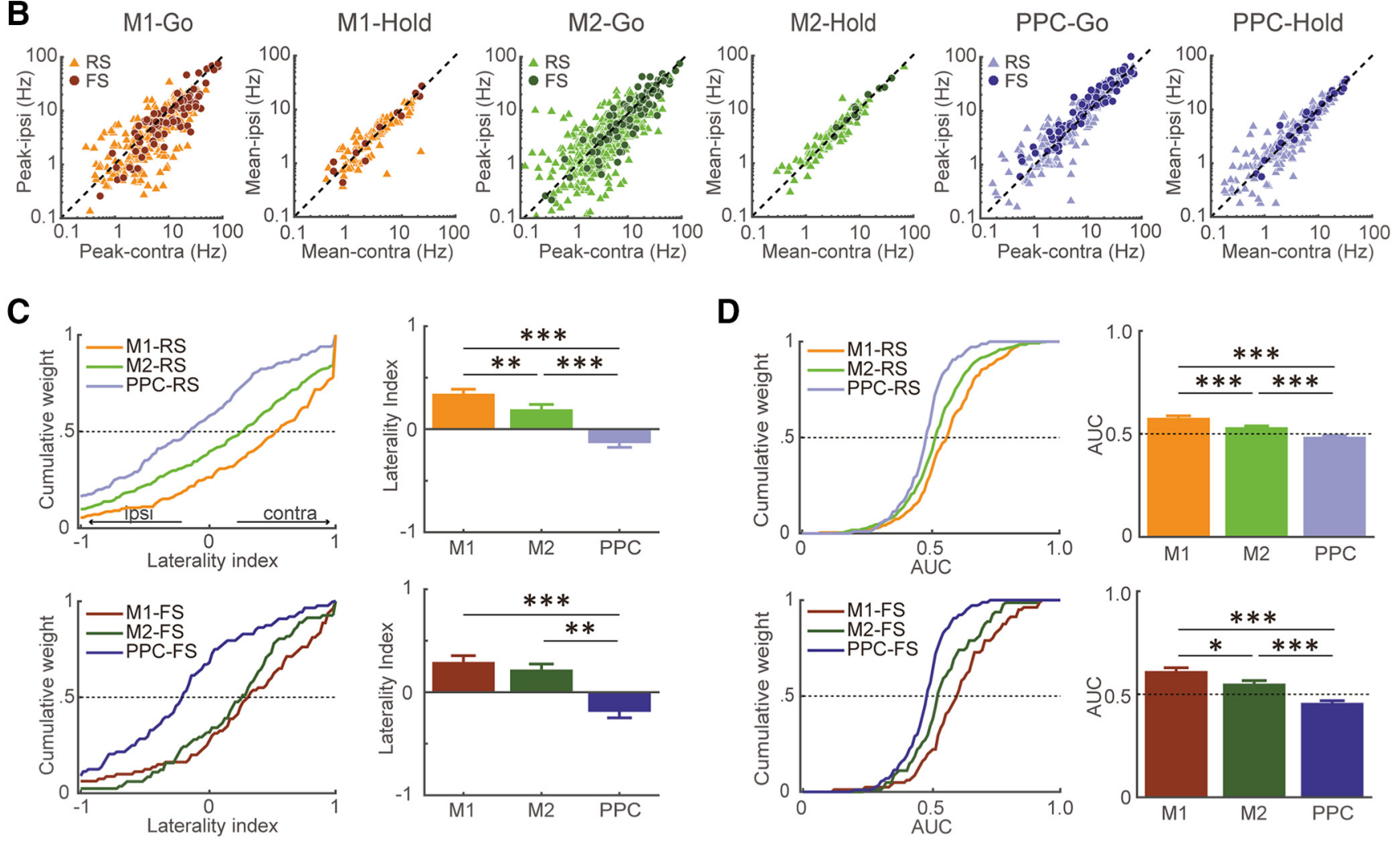

Figure 5. Preference of motor cortex and PPC neurons for contralateral or ipsilateral forelimb movements. A, Functional spike activity of RS and $F S$ subtypes of M1, M2, and PPC neurons for contralateral and ipsilateral forelimb movements. Each row shows normalized Gaussian-filtered PETH of a single neuron [aligned with the pedal release onset at $0 \mathrm{~s}$ (vertical line); $\sigma=12.5 \mathrm{~ms}$ for spikes in $0.05 \mathrm{~ms}$ bins] during contralateral and ipsilateral release trials. The task-related neurons were sorted by the order of peak time (early to late). $\boldsymbol{B}$, Distributions for the contralateral (abscissa) and ipsilateral (ordinate) release trials in peak activity of go-type neurons and in sustained activity of hold-type neurons in M1, M2, and PPC. Light triangles and dark circles represent individual RS and FS neurons, respectively. C, Cumulative distributions (left) and averages (right) of the laterality index of go-type activity for the RS (top) and FS (bottom) subtypes of M1, M2, and PPC neurons. Its positive and negative values indicate preference for contralateral and ipsilateral forelimb movements, respectively. Error bars indicate SEM. D, Cumulative distributions (left) and averages (right) of the AUCin ROC analysis between contralateral and ipsilateral trials of go-type activity for the RS (top) and FS (bottom) subtypes of M1, M2, and PPC neurons. See Materials and Methods for details. ${ }^{*} p<0.05$, ${ }^{* *} p<0.01,{ }^{* * *} p<0.001$, post hoc Scheffe's test.

ments (PPC-RS: $-0.14 \pm 0.62, z=-2.78, p<5.5 \times 10^{-3}$; PPC-FS: $\left.-0.19 \pm 0.52, z=-3.41, p<6.4 \times 10^{-4}\right)$. M1-RS and PPC-RS neurons represented more contralaterally and ipsilaterally biased forelimb movements, respectively, compared with
M2-RS neurons (two-way ANOVA, areas, $F_{(2,1034)}=34.8, p<$ $4.7 \times 10^{-15}$; post hoc Tukey's HSD test, M1 vs PPC, M2 vs PPC, $p<1.0 \times 10^{-3} ; \mathrm{M} 1$ vs M2, $\left.p<1.0 \times 10^{-2}\right)$. For FS neurons, we observed a similar tendency between the motor cortices and PPC 
(M1 vs PPC, M2 vs PPC, $p<1.0 \times 10^{-3}$; M1 vs $M 2, p=0.45)$. We observed no significant difference of laterality indices between RS and FS neurons (two-way ANOVA, cell types, $F_{(2,1034)}=0.09, p=$ $0.76)$. We also checked the laterality index in the baseline period $(-1000$ to $-700 \mathrm{~ms}$ from the pedal release onset). The PPC showed no biased activity in the baseline period (PPC-RS: $-0.01 \pm 0.19$, onesample signed-rank test, $z=0.27, p=$ 0.79; M2-FS: $-0.00 \pm 0.14, z=0.51, p=$ 0.61 ), although the motor cortices showed slight ipsilateral biases (M1-RS: $-0.03 \pm$ $0.24, z=-1.99, p<4.6 \times 10^{-2}$; M1-FS: $-0.03 \pm 0.17, z=-2.01, p<4.4 \times 10^{-2}$; M2-RS: $-0.03 \pm 0.27, z=-2.28, p<$ $2.3 \times 10^{-2} ; \mathrm{M} 2-\mathrm{FS}:-0.02 \pm 0.14, z=$ $-1.46, p=0.14)$.

We also evaluated the limb preference of go-type neurons using ROC analysis (Fig. 5D; see Materials and Methods). The AUCs of M1 and M2 neurons were significantly biased toward a preference for contralateral movements (M1-RS: $0.58 \pm 0.15$, one-sample signed-rank test, $z=7.03, p<2.1 \times 10^{-12}$; M1-FS: $0.61 \pm 0.15, z=5.83, p<5.5 \times 10^{-9}$; M2-RS: $0.53 \pm 0.13, z=$ 4.95, $p<7.4 \times 10^{-7}$; M2-FS: $0.55 \pm 0.13, z=3.32, p<9.1 \times$ $\left.10^{-4}\right)$. In contrast, the AUC of PPC neurons, both the RS and FS subtypes, were biased to ipsilateral movements (PPC-RS: $0.49 \pm$ $0.09, z=-2.218 p<2.7 \times 10^{-2}$; PPC-FS: $0.46 \pm 0.1, z=-3.71$, $\left.p<2.0 \times 10^{-4}\right)$. Consistent with the above results using the laterality index, M1 and PPC neurons represented more contralaterally and ipsilaterally biased forelimb movements, respectively, compared with M2 neurons (two-way ANOVA, areas: $F_{(2,1034)}=53.29, p<9.4 \times 10^{-23} ;$ post hoc Tukey's HSD test, $p<$ $1.0 \times 10^{-3}$ for all pairs of M1-RS, M2-RS, and PPC-RS; M1-FS vs PPC-FS, M2-FS vs PPC,-FS $p<1.0 \times 10^{-3}$; M1 vs M2, $\left.p<0.05\right)$. We again observed no difference of AUC between RS and FS neurons $\left(F_{(2,1034)}=0.92, p=0.34\right)$.

The PPC is known to be involved in attentional processes such as sensory history and evidence accumulation (Erlich et al., 2015; Hanks et al., 2015; Scott et al., 2017; Akrami et al., 2018). Therefore, the biased functional activity could be modulated around the rule reversal (i.e., block changes; cf. Fig. $1 B$ ) where a conflict might occur between attention to the right and left limbs. We therefore investigated whether the biased functional activity was transiently affected by block changes (Fig. 6; see Materials and Methods). For this analysis, we examined the fractions of go-type neurons that showed remarkable limb preference (laterality in$\operatorname{dex}>0.5$ or $<-0.5$; M1-RS, contralateral $n=107$, ipsilateral $n=$ 28; M2-RS, contralateral $n=165$, ipsilateral $n=84$; PPC-RS, contralateral $n=28$, ipsilateral $n=56$ ). We found no significant modulation of functional activity among trial numbers after block changes in these cortical areas (two-way ANOVA; trials: contralateral, $F_{(9,1658)}=1.07, p=0.38$; ipsilateral, $F_{(9,1587)}=$ $1.60, p=0.11$; areas: contralateral, $F_{(9,1658)}=0.58, p=0.56$; ipsilateral, $\left.F_{(2,1587)}=0.81, p=0.45\right)$. There was no detectable modulation of functional activity by a possible attentional conflict between right and left forelimb controls.

\section{Forelimb movements evoked by optogenetic activation of motor cortices and PPC}

The above observations suggest that the PPC, unlike the motor cortices, may participate in ipsilaterally biased forelimb move- ments. To test this hypothesis, we attempted to reproduce muscular movements by optogenetic activation of these cortical areas using Thy1-ChR2 transgenic rats, whose cortical neurons abundantly expressed ChR2-Venus under the control of the Thy1promotor (W-TChR2V4; Tomita et al., 2009; Saiki et al., 2018). We stimulated the surface of the motor cortices or PPC by blue light (50 pulses at $20 \mathrm{~Hz} ; 2 \mathrm{~ms}$ pulse duration) while the rats held both pedals continuously (Fig. 7, and Movie 1). Figure 7A shows averaged changes in pedal position triggered by optogenetic cortical activation. The optogenetic activation of M1 and M2 forelimb areas evoked a large pedal release preferentially on the contralateral side, whereas PPC activation evoked a release on the ipsilateral side. The laterality index of pedal position change revealed contralateral- and ipsilateral-dominant pedal releases by the optogenetic activation of motor cortices and PPC, respectively (Fig. $7 B$, top; M1: $n=11$ rats, mean \pm SEM; $0.97 \pm 0.08$, one-sample signed-rank test, $z=3.18, p<2.4 \times 10^{-4}$; M2: $n=$ 11 rats, $0.48 \pm 0.78, z=2.48, p<1.0 \times 10^{-2}$; PPC: $n=16$ rats, $-0.70 \pm 0.64, z=-3.43, p<5.9 \times 10^{-4}$; see also Materials and Methods). The evoked changes in pedal position differed significantly between the motor cortices and PPC (Kruskal-Wallis test, $H=27.80, p<9.2 \times 10^{-7}$; post hoc Scheffe's test, M1 vs PPC, M2 vs PPC, $p<1.0 \times 10^{-3}$ ). We also analyzed the latency of pedal release on the preferred side (contralateral for M1 and M2; ipsilateral for PPC), and observed that optogenetic M1 activation evoked significantly faster pedal releases than PPC activation (Fig. 7B, bottom; M1, $137 \pm 83 \mathrm{~ms}$; M2, $246 \pm 142 \mathrm{~ms}$; PPC, $267 \pm 131 \mathrm{~ms}$; Kruskal-Wallis test, $H=7.03, p<3.0 \times 10^{-2}$; post hoc Scheffe's test, M1 vs PPC, $p<3.9 \times 10^{-2}$ ). Similarly, the latency of subsequent push down was shorter in M1 activation than in PPC activation (M1, $948 \pm 1000 \mathrm{~ms}$; M2, $1059 \pm 997 \mathrm{~ms}$; PPC, $\left.1510 \pm 864 \mathrm{~ms} ; H=6.53, p<3.8 \times 10^{-2}\right)$. The pedal release durations tended to be longer in PPC activation than in M1 activation between cortical area-specific activation (M1, $384 \pm 620 \mathrm{~ms} ; \mathrm{M} 2,440 \pm 565 \mathrm{~ms}$; PPC, $705 \pm 710 \mathrm{~ms} ; H=3.67$, $p=0.16)$.

Although muscular activity was well correlated with changes in pedal position (as illustrated in Fig. 1C), the pedal position could not be suitable to detect certain forelimb movements, for instance, pushing the pedal further down. Therefore, we tried to detect forelimb movements themselves in video analysis (Fig. $7 C, D$; see Materials and Methods). Figure $7 C$ (top) shows pseudocolored images of changes in the value of each pixel, which reflect forelimb deviation by optogenetic activation of M1, M2, and PPC in a representative session. The forelimb deviation on 
A

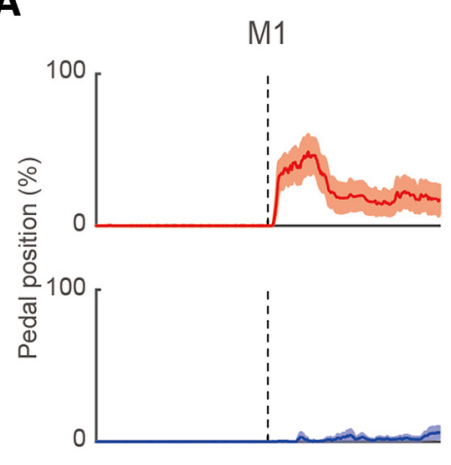

C
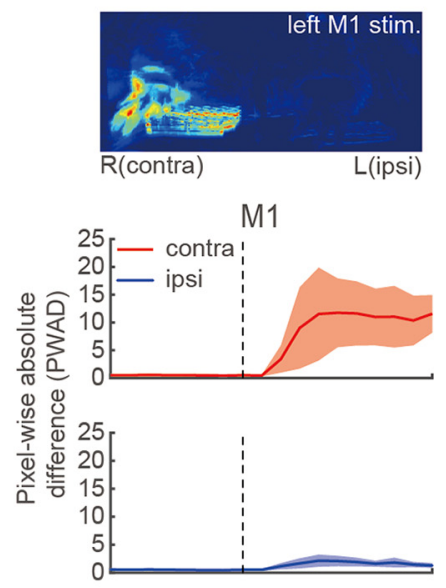

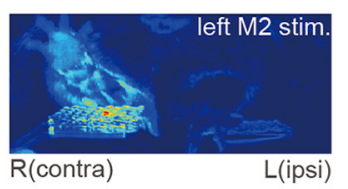

M2

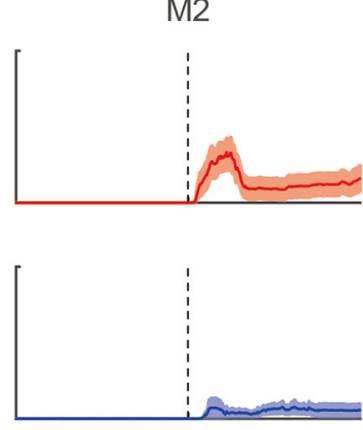

M2

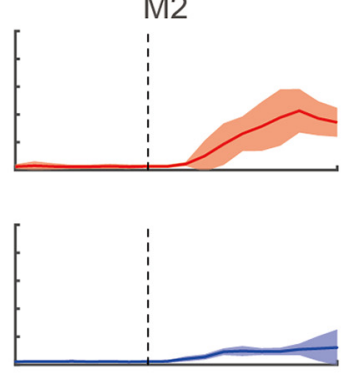

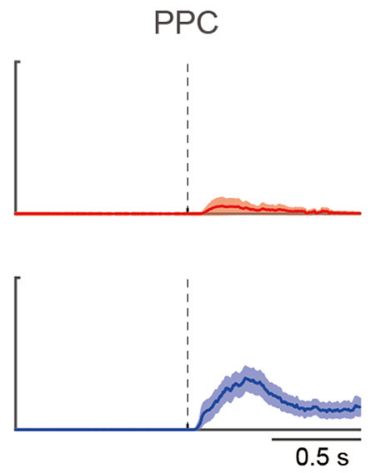

$0.5 \mathrm{~s}$

\section{D}
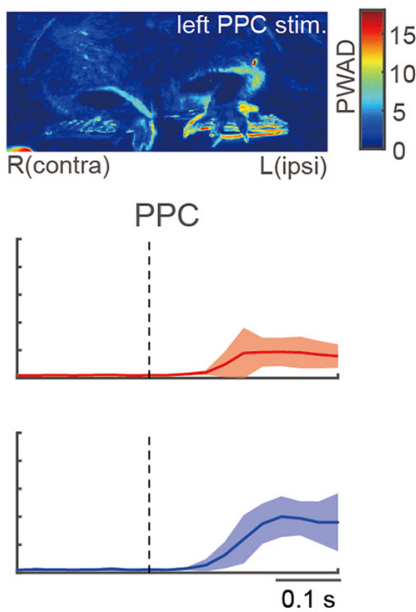

B

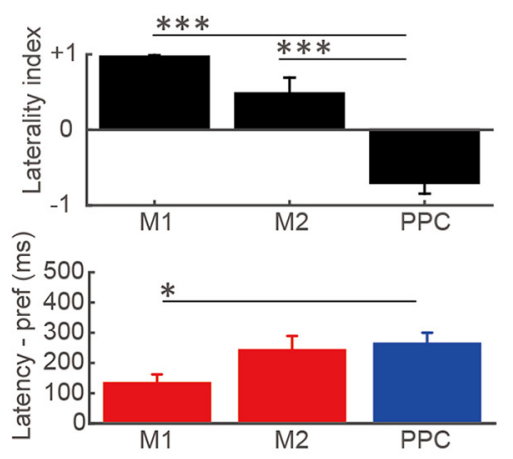

Figure 7. Forelimb movements evoked by optogenetic activation of motor cortices and PPC. $\boldsymbol{A}$, Temporal changes in contralateral (red) and ipsilateral (blue) pedal position (mean \pm SEM) evoked by optogenetic activation (dashed line) of M1, M2, and PPC in ChR2-expressing Thy1-ChR2 rats (M1: $n=11, \mathrm{M} 2: n=11$, PPC: $n=16$ ). $\boldsymbol{B}$, Laterality index (top) and latency (bottom) of the pedal position change (mean \pm SEM) evoked by optogenetic activation. The latency was measured on the preferred side determined by the laterality index (contralateral for M1 and M2; ipsilateral for PPC). C, Video analysis of forelimb movements by the same optogenetic activation. Top, Representative images of PWAD evoked by optogenetic activation of the left M1, M2, and PPC. Bottom, Temporal changes in PWAD for contralateral (red) and ipsilateral (blue) forelimbs. $\boldsymbol{D}$, Laterality index of forelimb deviation in PWAD evoked by optogenetic activation. $\boldsymbol{E}$, Forelimb movements evoked by optogenetic M1 and PPC activation in a pedal-free (dangling) condition in several rats $(n=5)$. 0 pen and filled circles represent M1 and PPC stimulation, respectively, each of which was plotted by the PWAD values for contralateral (abscissa) and ipsilateral (ordinate) forelimbs. ${ }^{*} p<0.05,{ }^{* * *} p<0.001$; Kruskal-Wallis test with post hoc Scheffe's test.

each side was quantified by calculating the PWAD (see Materials and Methods; Powell et al., 2015; Goard et al., 2016) in all nine rats examined (Fig. 7C, bottom). Optogenetic M1 and M2 activation caused forelimb deviation preferentially on the contralateral side, whereas optogenetic PPC activation caused bilateral forelimb deviation that was stronger on the ipsilateral side. Similar observations were obtained when cortical areas were electrically stimulated using a standard ICMS method (data not shown).

The laterality index in PWAD revealed the contralateral and ipsilateral dominance of forelimb movements evoked by optogenetic activation of motor cortices and PPC, respectively (Fig. 7D; mean \pm SEM; M1: $0.77 \pm 0.08$, one-sample signed-rank test, $z=$ 2.67, $p<3.9 \times 10^{-2} ; \mathrm{M} 2: 0.51 \pm 0.20, z=2.67, p<3.9 \times 10^{-2}$; PPC: $-0.35 \pm 0.31, z=-2.40, p<1.4 \times 10^{-2}$ ), which was consistent with the results of pedal trace analysis (Fig. $7 B$ ). The laterality indices in PWAD were significantly different between the motor cortices and PPC (Kruskal-Wallis test, $H=34.06, p<$ $1.9 \times 10^{-5}$; post hoc Scheffe's test, M1 vs PPC, $p<1.0 \times 10^{-3}$; $\mathrm{M} 2$ vs PPC, $p<0.05)$. We note that PPC activation often induced a "swing-like" movement of the ipsilateral forelimb (Movie 1; see Discussion).
It is possible that the presence of pedals restricted downward movements of the forelimbs (i.e., standing firm) during optogenetic activation. Therefore, we removed the pedals from the task device to keep the forelimbs free to move in several rats and obtained similar results (Fig. 7E; M1, $n=55$ trials; PPC, $n=53$ trials from 5 rats); in other words, optogenetic PPC activation caused ipsilaterally biased forelimb movements in contrast with optogenetic M1 and M2 activation.

\section{Mapping of forelimb movements evoked by optogenetic PPC activation}

As part of the association cortex, the PPC processes diverse sensory- and motor-related information (Taira et al., 1990; Gallese et al., 1994; Sakata et al., 1995; Chang et al., 2008; Chang and Snyder, 2012; Goard et al., 2016; Scott et al., 2017; Akrami et al., 2018; Yoshida et al., 2018). Therefore, it is possible that the activated site of the PPC caused ipsilaterally biased movements only locally. Therefore, we mapped the laterality index of forelimb movements evoked by grid-like optogenetic activations of the PPC and adjacent areas in awake rats (500 $\mu \mathrm{m}$ intervals; Hira et al., 2015), which was measured by video analysis using PWAD (Fig. $8 A ; n=4$ rats; Powell et al., 2015; Goard et al., 2016). The 
Unilateral photoactivation Left M1 in the W-TChR2V4
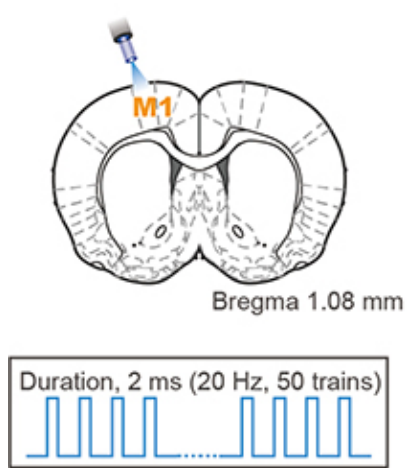

Right forelimb: Contralateral Left forelimb: Ipsilateral

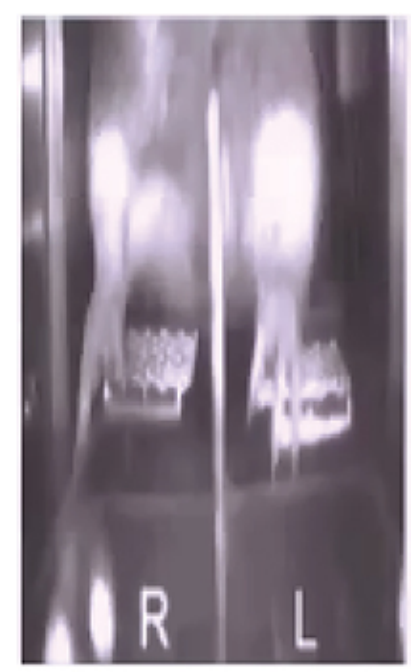

Movie 1. Forelimb movements evoked by optogenetic activation of M1 and PPC in a Thy1-ChR2 rat. A pulse of blue LED light was applied unilaterally (e.g., left hemisphere) to the M1 or PPC. Forelimb movements were analyzed by using both pedal trajectories and video data (see Materials and Methods).

optogenetic activation (50 pulses at $20 \mathrm{~Hz} ; 2 \mathrm{~ms}$ pulse duration) at each site of the PPC (green rectangle; cf. Fig. $2 B, C$ ) evoked bilateral forelimb movements that were biased more ipsilaterally than contralaterally (Fig. 8B). Therefore, the whole PPC had the potential to express ipsilateral forelimb movements. Anteriorly, optogenetic activation of the motor cortex (medial) and somatosensory cortex (lateral) primarily evoked contralateral forelimb movements (top right corner). Posteriorly, we observed no significant forelimb movements evoked by optogenetic activation of the visual cortex (bottom right corner). Mapping studies of motor responses by PPC stimulation have been reported in monkeys (Stepniewska et al., 2005, 2009; Gharbawie et al., 2011a,b; Kaas and Stepniewska, 2016) and tree shrews (Baldwin et al., 2017). In rodents, however, motor responses of forelimbs to cortical stimuli have only been mapped for the frontal motor cortices (Donoghue and Wise, 1982; Neafsey et al., 1986; Deffeyes et al., 2015; Hira et al., 2015). Our report is likely the first mapping study of cortically evoked forelimb movements in the rodent PPC.

\section{Effects of optogenetic PPC inhibition via GABA release on task performance}

Our results suggest that the artificial activation of the PPC was sufficient to induce ipsilaterally biased movements in two forelimbs (Figs. 7, 8). Next, we investigated whether the PPC is involved in contralateral and ipsilateral forelimb movements to correctly release the pedal during task performance. To inhibit cortical activity, inhibitory GABAergic interneurons expressing ChRWR-Venus were optogenetically excited by local blue light illumination (Fig. 9; optogenetic inhibition via GABA release). For this purpose, we combined the VGAT-Cre transgenic rats expressing Cre recombinase under the control of the VGAT promotor (Igarashi et al., 2018) with AAV vector which expresses the ChRWR-Venus gene under the control of CAGGS promoter in a Cre-dependent manner (AAV-CAGGS-DIO-ChRWR-Venus vector; see Materials and Methods). In a preliminary experiment, we histologically confirmed the specificity of Cre-dependent gene expression to GABAergic interneurons in the cerebral cortex of VGAT-Cre rats ( $n=2$ rats; Fig. $9 A$ ) that were crossed with
tdTomato reporter rats (Igarashi et al., 2016). Furthermore, we recorded a total of 28 neurons in ex vivo slices from 3 VGAT-cre rats that received virus injections in the PPC and M1. We recorded one and two Venus ${ }^{+}$cells from the right and left PPC, respectively, and one and three Venus ${ }^{+}$cells from the right and left M1, respectively. All 7 neurons responded to blue light stimulation with robust and stable ChRWRmediated inward currents (data not shown). Also, we recorded 6 and 4 Venus ${ }^{-}$cells from the right and left PPC, respectively, and 7 and 4 Venus ${ }^{-}$cells from the right and left M1, respectively. All 21 neurons responded to blue light stimulation with robust and stable $\mathrm{GABA}_{\mathrm{A}}$ receptor-mediated postsynaptic currents that were abolished by bicuculline (Fig. $9 B$ ). We also electrophysiologically confirmed the inhibitory effect of optogenetic excitation of ChRWR-expressing GABAergic neurons on nearby recorded neurons in the viral vector-infected cortical areas in vivo (Fig. 9C,D). Most of the recorded PPC neurons near the injection site effectively decreased their spike rate during blue light illumination; in other words, optogenetic GABA release inhibited spiking (Fig. $9 D$, right; $n=26$ neurons, Friedman test, $\chi^{2}=41.77, p<8.6 \times 10^{-10}$; post hoc Scheffe's test, $p<1.0 \times 10^{-3}$ for stimulation vs pre- or poststimulation).

While the rats performed the right-left pedal task, unilateral M1 or PPC inhibition by optogenetic GABA release was conducted around the time expected for pedal release $(0.5-1.5 \mathrm{~s}$ after hold start) in randomly chosen trials (10 trials each for control and test) in the middle of contralateral- and ipsilateral-rewarded blocks (Fig. 9E; also see Materials and Methods). We observed that optogenetic PPC inhibition tended to increase incorrect releases of the contralateral pedal in the ipsilateral-rewarded blocks (PPC-ipsi, OFF $97.4 \pm 3.6 \%$ vs ON $95.0 \pm 4.3 \%$, signed-rank test, $z=-1.72, p=0.09$ ), whereas rats performed correctly in the control (no optogenetic inhibition) trials as well as in test and control trials of the contralateral-rewarded blocks (PPC-contra, OFF $98.0 \pm 2.3 \%$ vs ON $97.7 \pm 2.5 \%, z=-0.14, p=0.90$; Fig. $9 E$, right). Conversely, optogenetic M1 inhibition showed contrasting effect to PPC inhibition; that is, increase in incorrect releases of the contralateral pedal (M1-contra, OFF $92.3 \pm 5.8 \%$ vs ON 90.6 $\pm 7.7 \%, z=0.82, p=0.41$; M1-ipsi, OFF $95.6 \pm 2.3 \%$ vs ON $96.7 \pm 1.9 \%, z=2.56, p<1.1 \times 10^{-2}$ ). These effects were quantified by calculating the net effect of optogenetic cortical inhibition, in which positive and negative values indicate a shift of limb preference toward the contralateral and ipsilateral side, respectively (see Materials and Methods). The net effect of optogenetic PPC inhibition was significantly positive compared with the chance level; that is, the limb preference shifted contralaterally (Fig. $9 F ; n=4$ rats, 12 sessions; one-sample signed-rank test, $\left.z=-2.31, p<1.9 \times 10^{-2}\right)$. Conversely, optogenetic M1 inhibition significantly shifted the limb preference ipsilaterally $(z=$ 2.40, $p<1.4 \times 10^{-2}$ ). The contrasting shifts between M1 and PPC were also statistically significant (Mann-Whitney test, $z=$ $\left.3.09, p<2.0 \times 10^{-3}\right)$.

We found no significant change in holding time via optogenetic M1 and PPC inhibition [Fig. 9G,H; median (interquartile range); M1: contralateral-rewarded block, ON vs OFF, 2.0 (1.7$2.7 \mathrm{~s})$ vs $1.9(1.7-2.6 \mathrm{~s})$, Mann-Whitney test, $z=0.91, p=0.36$; ipsilateral, 2.0 (1.7-2.8 s) vs 2.0 (1.7-2.7 s), $z=1.45, p=0.15$; PPC: contralateral, $2.4(1.9-2.8 \mathrm{~s})$ vs $2.5(1.9-2.8 \mathrm{~s}), z=-1.11$, $p=0.27$, ipsilateral, $2.2(1.9-2.7 \mathrm{~s})$ vs $2.3(1.9-2.7 \mathrm{~s}), z=-0.93$, $p=0.35]$, suggesting that optogenetic cortical inhibition altered the forelimb preference per se without affecting the execution (initiation) of forelimb movements. 
A

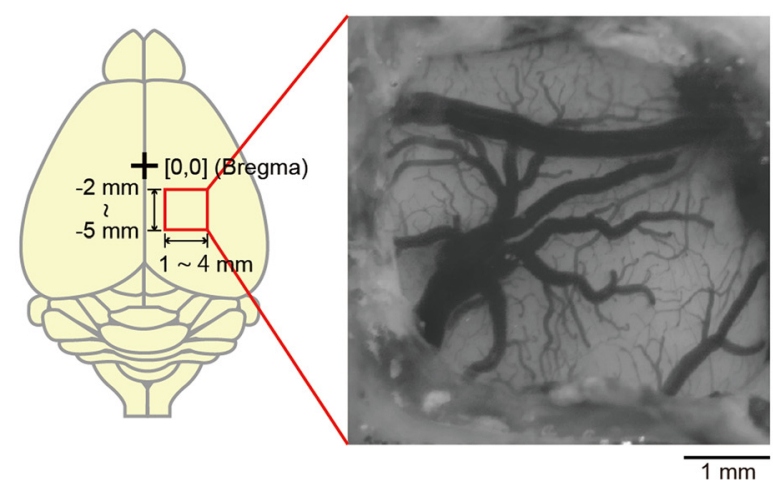

B

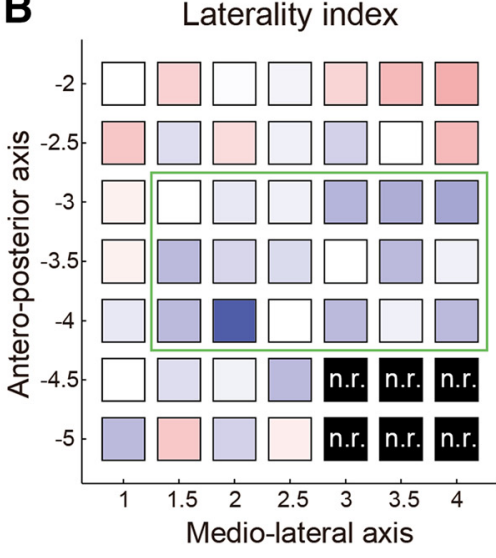

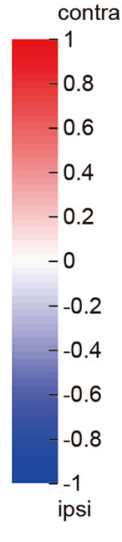

Figure 8. Mapping of forelimb movements evoked by optogenetic PPC activation. $A$, Optogenetic activation in and around the PPC (AP: -2.0 to $-5.0 \mathrm{~mm}$; ML: $1.0-4.0 \mathrm{~mm}$, every $0.5 \mathrm{~mm}$ in a grid-like manner) to map evoked forelimb movements and preference. $\boldsymbol{B}$, Forelimb preference map based on laterality index ( $n=4$ rats). The laterality index is color-coded as follows: blue, toward -1 (ipsilateral); white, 0 (bilateral); red, toward +1 (contralateral). Black indicates nonresponsive (n.r.) in all rats. Green rectangle indicates the putative PPC area (Fig. 2B,C).

\section{Effects of weak optogenetic PPC activation on task performance}

It is likely that the PPC processes abstract information for motor planning and preparation rather than actual motor execution (Snyder et al., 1997, 1998; Shadlen and Newsome, 2001). Given that PPC activity represents bilateral limb movements, the PPC might abstractly coordinate motor planning and preparation for optimal movements of contralateral and ipsilateral limbs. Therefore, we tested whether the behavioral performance requiring abstract bilateral coordination could be affected by weak optogenetic cortical activation that alone was insufficient in eliciting any motor response in Thy1-ChR2 rats (Fig. 10). The intensity of optogenetic activation was weakened by decreasing the duration of light pulse (10 or $50 \mu \mathrm{s}$; cf. originally $2 \mathrm{~ms}$ in Figs. 7, 8) and the number of pulses ( 10 pulses at $20 \mathrm{~Hz}$; originally 50 pulses) so that the weak optogenetic activation by itself evoked almost no forelimb movements in PWAD (Fig. $10 A ; n=5$ rats; M1-contra: Mann-Whitney test, $10 \mu \mathrm{s}$ vs $50 \mu \mathrm{s}, z=-1.36, p=0.18$; $10 \mu \mathrm{s}$ vs $100 \mu$ s, $\left.z=-2.20, p<8.76 \times 10^{-3}\right)$. Each pulse of the weak optogenetic activation readily elicited cortical spike activity (Fig. 10B; Mann-Whitney test, $n=119$ neurons; $z=4.41, p=1.1 \times$ $\left.10^{-5}\right)$.

While the rats performed the behavioral task, weak optogenetic activation was unilaterally applied to the M1 or PPC around the time expected for pedal release (see Materials and Methods) and its net effect was calculated for M1 and PPC activation (Fig. $10 \mathrm{C}$ ). Although weak optogenetic M1 activation had no effect on limb preference during task performance, the weak PPC activation significantly shifted the limb preference ipsilaterally $(n=4$ rats, 12 sessions; M1: contralateral, OFF $90.3 \pm 7.0 \%$ vs ON $90.3 \pm 6.9 \%$, signed-rank test, $z=-0.71, p=0.51$; ipsilateral, OFF $93.9 \pm 4.4 \%$ vs ON $93.5 \pm 4.5 \%, z=-0.25, p=0.81$; one-sample signed-rank test, $z=-0.10, p=0.92$; PPC: contralateral, OFF $93.6 \pm 5.4 \%$ vs ON $92.3 \pm 6.6 \%, z=-1.44, p=$ 0.16; ipsilateral, OFF $93.2 \pm 7.2 \%$ vs ON $95.4 \pm 5.7 \%, z=-2.49$, $p<9.8 \times 10^{-3} ; z=-2.67, p<4.9 \times 10^{-3} ;$ Mann-Whitney test, $z=2.52, p<1.2 \times 10^{-2}$ between M1 and PPC). Importantly, no contralaterally biased forelimb movements were evoked by any milder range of optogenetic PPC activation than the original (Fig. $10 A)$. It is therefore unlikely that the original PPC activation (Figs. 7,8 ) was so strong as to disrupt its possible contralateral preference. The weak optogenetic activation had no effect on holding time [median (interquartile range); $\mathrm{M} 1$ : contralateralrewarded block, ON vs OFF, 2.5 (1.7-3.5 s) vs 2.2 (1.7-3.7 s), Mann-Whitney test, $z=-0.72, p=0.47$; ipsilateral, $2.2(1.8-3.2$ s) vs $2.2(1.7-3.2 \mathrm{~s}), z=0.10, p=0.92$; PPC: contralateral, 2.0 $(1.7-3.0 \mathrm{~s})$ vs $2.1(1.7-3.2 \mathrm{~s}), z=-0.73, p=0.47$, ipsilateral, 2.2 $(1.8-3.2 \mathrm{~s})$ vs $2.1(1.7-3.2 \mathrm{~s}), z=0.90, p=0.37]$. These observations support the idea that weak PPC activation affected abstract motor planning and preparation rather than downstream motor execution.

\section{Discussion}

To elucidate the functional preference of PPC neurons for contralateral and ipsilateral forelimb movements in rodents, we conducted electrophysiological recordings and optogenetic manipulations on PPC neurons in head-fixed rats performing the right-left pedal task (Fig. 1). Our main findings are summarized as follows: (1) the RS subtype of PPC neurons included more hold-type and fewer go-type activities than the FS subtype (Fig. 4D), which was similar with M1 and M2 neurons (Soma et al., 2017; Saiki et al., 2018); (2) the peak time distribution of go-type activity was faster in PPC neurons than in M2 neurons (Fig. 4E); (3) go-type PPC neurons preferentially represented ipsilateral, rather than contralateral, forelimb movements in contrast to M1 and M2 neurons (Fig. 5; see also Soma et al., 2017); (4) optogenetic PPC activation reproduced ipsilaterally biased forelimb movements in contrast to optogenetic M1 and M2 activation (Figs. 7, 8); (5) optogenetic PPC and M1 inhibition via GABA release caused a shift to contralateral and ipsilateral limb preference, respectively (Fig. 9); and (6) weak optogenetic PPC activation shifted the limb preference to ipsilateral without affecting motor initiation (Fig. 10). To the best of our knowledge, this is the first study to report the limb preference of parietal cortex neurons in rodents.

\section{Functional differences among rodent $\mathrm{M} 1, \mathrm{M} 2$, and PPC neurons}

In our previous and present studies, we classified functional (task-related) M1, M2, and PPC neurons into hold-type and gotype neurons according to peak time position and dependence on holding time (Fig. 4A-C; Soma et al., 2017). Judging from the behavioral correlation, we suggest that hold-type neurons could be involved in keeping the body parts still, whereas go-type neurons could be involved in motor execution itself or higher-order 
A
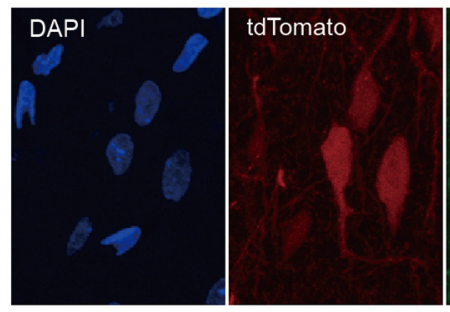

B
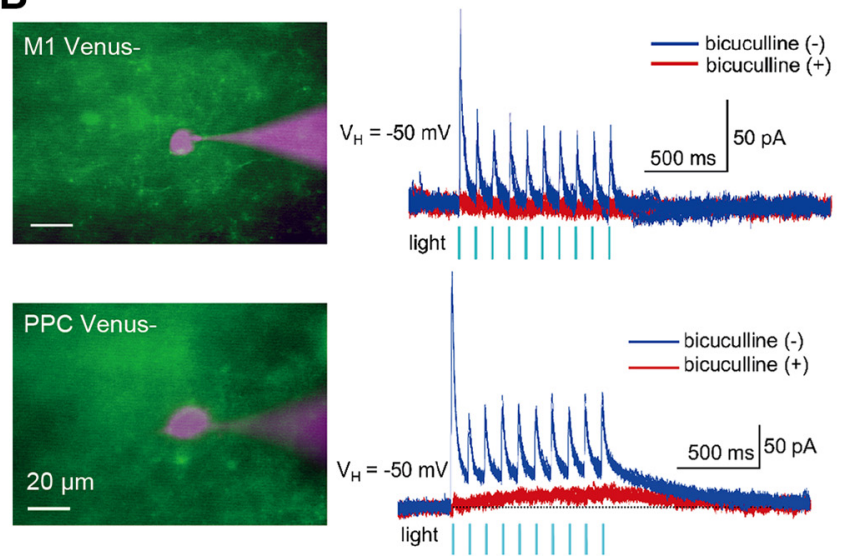

C
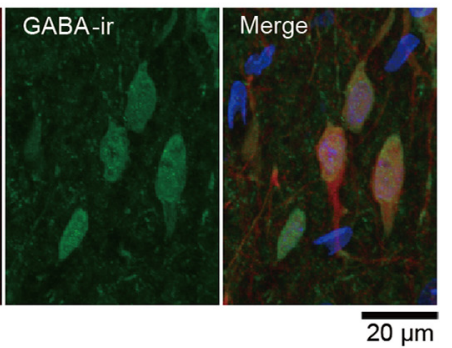

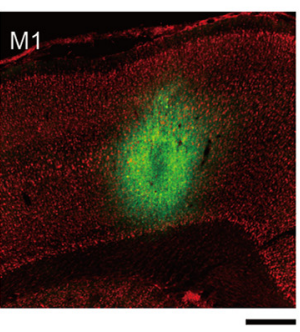

D

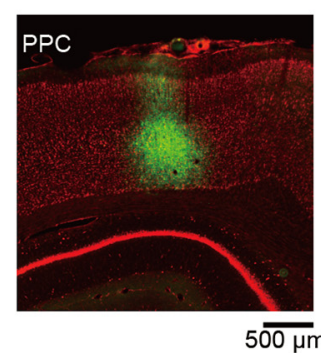

E

Unilateral optogenetic inhibition
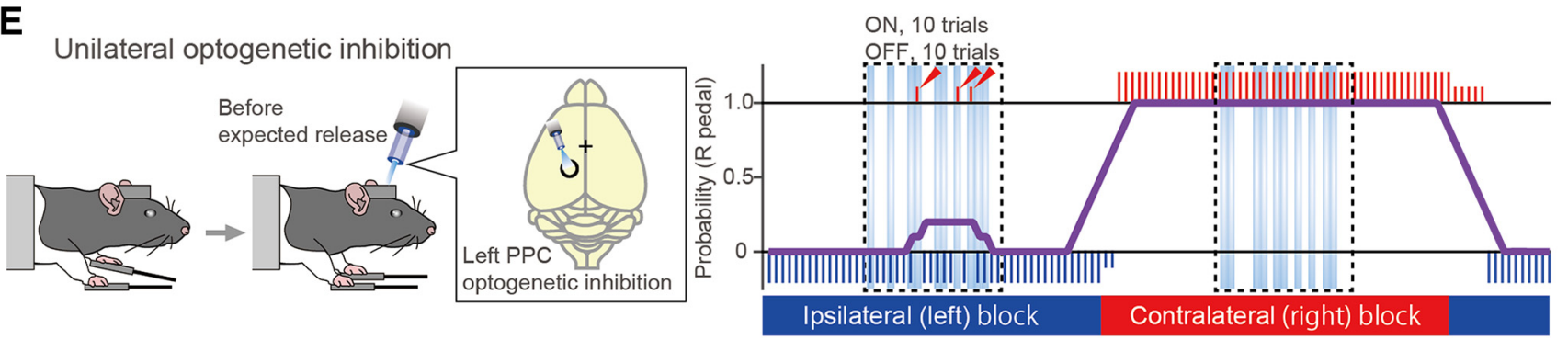

F

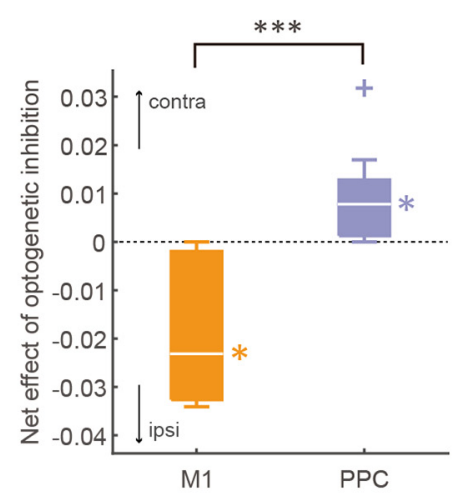

G
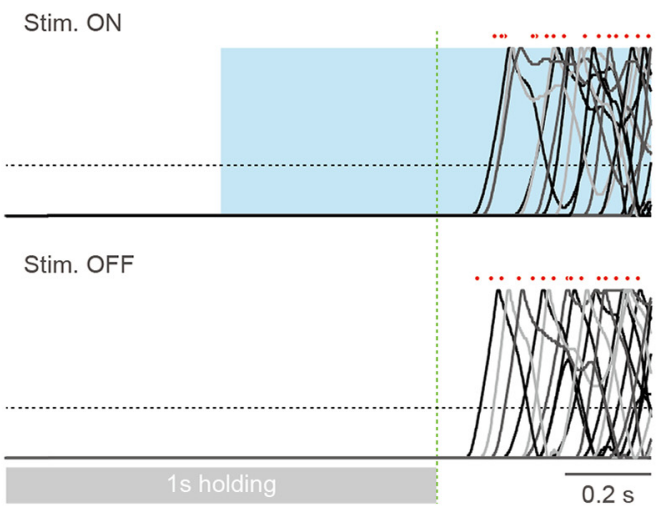

H
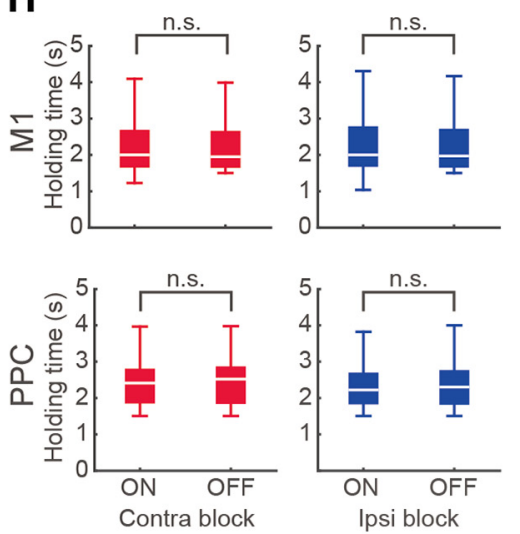

Figure 9. Effects of optogenetic M1 and PPC inhibition via GABA release on task performance. $A$, Cre-dependent gene expression specific to GABAergic interneurons in the M1 of a VGAT-Cre rat crossed with a tdTomato reporter rat. Colors show DAPI (blue), tdTomato (red), and GABA immunoreactive (green). B, Light-evoked responses of M1 (top) and PPC (bottom) neurons in slices obtained from VGAT-Cre rats that were infected with AAV vector (AAV-CAGGS-DI0-ChRWR-Venus vector) for Cre-dependent expression of ChR-wide receiver (ChRWR)-Venus. Left, Overlaid images of Venus fluorescence (green) and recorded neurons loaded with Alexa Fluor 594 (magenta). Right, Membrane currents in response to repeated light stimulation in the absence (blue) and presence (red) of bicuculline $(10 \mu \mathrm{M})$. The outward currents were abolished by bicuculline, strongly suggesting that they resulted from GABA release by optogenetic excitation of inhibitory interneurons. The Venus ${ }^{-}$neurons showing such postsynaptic responses never showed light-activated direct inward current. C, Expression of ChRWR-Venus (green) in the cortical area of VGAT-Cre rat after the virus injection. The sections were counterstained with Neurotrace (fluorescent Nissl stain; red). $D$, An example of cortical neuron (left) and statistical data of tested neurons (right, $n=26$ ) showing spike activity was suppressed under GABA release by optogenetic excitation of interneurons. ${ }^{* * *} p<0.001$; Friedman test with post hoc Scheffe's test. $\boldsymbol{E}$, Optogenetic PPC inhibition via GABA release during task performance. Left, Unilateral optogenetic PPC inhibition (for $1 \mathrm{~s}$ ) around the expected timing of pedal release (see Materials and Methods). Right, Representative performance through several blocks. In the performance diagram, long and short vertical bars (red, right release; blue, left release) represent rewarded and nonrewarded trials, respectively. Purple line represents the probability of right pedal release in past 10 trials. Ten test $(\mathrm{ON}$, optogenetic inhibition) and 10 control (OFF, no optogenetic inhibition) trials were randomly arranged (Figure legend continues.) 
A

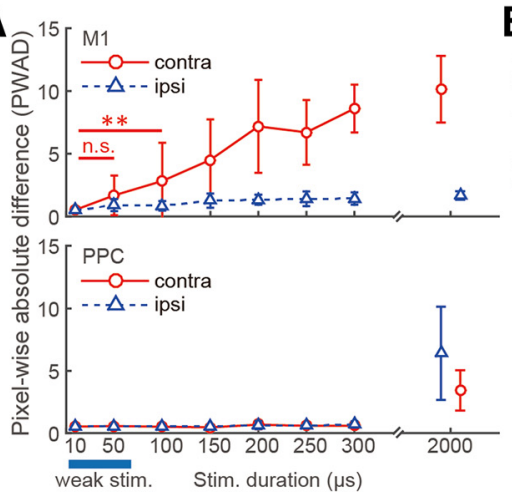

B

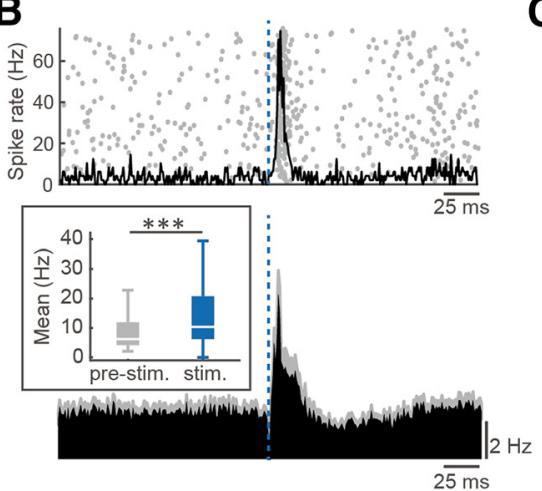

c

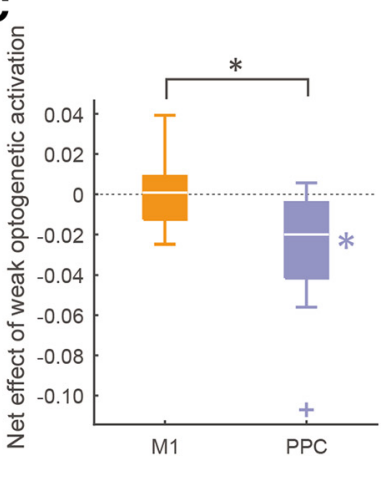

Figure 10. Effects of weak optogenetic M1 and PPC activation on task performance. $\boldsymbol{A}$, Preliminary data to determine the stimulus intensity (adjusting with the duration) that evoked no motor response by itself. Evoked motor response of each forelimb (in PWAD) was plotted against the stimulus duration in optogenetic M1 (top) and PPC (bottom) activation. We used a short stimulus duration (blue bar; 10 or $50 \mu \mathrm{s}$, cf. originally, $2 \mathrm{~ms}$ ) that evoked almost no motor response in the weak optogenetic activation experiments (see below in $\boldsymbol{C}$ ). ${ }^{* *} p<0.01$, Mann-Whitney test. $\boldsymbol{B}$, Preliminary data to confirm that the weak optogenetic cortical activation readily elicited spikes in nearby neurons in the ChR2-expressing Thy1-ChR2 rats. Top, Representative M1 neuron showing spike increase (raster plot and PETH) after weak optogenetic M1 activation (blue dashed line). Bottom, Averaged PETHs (with SEM) in all tested neurons ( $n=119$ ). Inset, Comparison of mean spike rate between baseline (pre-stimulation., -50 to 0 ms) and immediately after the optogenetic activation (stimulation, 0 to 50 ms). ${ }^{* * *} p<0.001$, Mann-Whitney test. $\boldsymbol{C}$, Net effects of weak optogenetic M1 and PPC activation on task performance. Notations are as in Figure $9 F .{ }^{*} p<0.05$, one-sample signed-rank test (within area), Mann-Whitney test (between areas).

motor function such as motor planning and preparation. We demonstrated that only go-type neurons displayed laterally biased limb preference in the three areas: M1 neurons preferred the contralateral limb and M2 and PPC neurons preferred both limbs, but were biased contralaterally and ipsilaterally, respectively (Fig. 5). The difference in limb preference between M1 and $\mathrm{M} 2$ neurons in rodents is comparable to that among the M1, PM, and SMA neurons in primates (Tanji et al., 1987; Cisek et al., 2003; Kurata, 2010; Nakayama et al., 2015). Functionally, rodent M1 neurons directly contribute to motor execution, whereas M2 neurons seem to be associated with motor planning and preparation rather than the motor execution (Narayanan and Laubach, 2009; Li et al., 2016); this is similar to M1 and PM/SMA neurons in primates (Weinrich and Wise, 1982; Mushiake et al., 1991; Hoshi and Tanji, 2000; Churchland et al., 2006; Shenoy et al., 2013). Likewise, rodent PPC neurons seemed to be associated with motor planning and preparation (as demonstrated in Fig. 10 ), which is also similar to primate PPC neurons (Snyder et al., 1997, 1998, 2000; Shadlen and Newsome, 2001; Quian Quiroga et al., 2006).

Ipsilaterally biased limb preference of rodent PPC neurons Primates have functionally differentiated motor cortices in the cerebral cortex; M1 neurons encode motor information on mostly contralateral body parts, whereas PM/SMA neurons encode higher-order information on bilateral, but still contralaterally biased, body movements (Evarts, 1966; Tanji et al., 1987; Kurata, 2010; Griffin et al., 2015; Nakayama et al., 2015). Similarly, primate PPC represents bilateral information with a bias to

$\leftarrow$

(Figure legend continued.) in the middle of each block (in the dashed rectangle). Arrowheads indicate incorrect (nonrewarded) pedal releases in the test trials. $\boldsymbol{F}$, Net effects of optogenetic M1 and PPC inhibition on task performance (toward -1 , shift to ipsilateral; +1 , contralateral; see Materials and Methods). Box plot shows the net effect of optogenetic inhibition (white bar, median; upper and lower edges, quartiles; whiskers, error; cross, outlier) for M1 and PPC. ${ }^{*} p<$ 0.05 , one-sample signed-rank test (within area), ${ }^{* * *} p<0.001$, Mann-Whitney test (between areas). $\mathbf{G}$, Representative pedal traces in the presence (top) and absence (bottom) of optogenetic cortical inhibition aligned with the time at $+1 \mathrm{~s}$ after hold start (green dashed line). Dots indicate the pedal release onset in each trial. $\boldsymbol{H}$, No effect of optogenetic cortical inhibition on holding time, which reflects motor initiation. contralateral (Kermadi et al., 2000; Chang et al., 2008; Chang and Snyder, 2012). Our previous study revealed that rodent M1 and M2 neurons also encode motor information that is dominant on the contralateral side, which is very similar to the primate M1 and PM/SMA neurons (Soma et al., 2017). However, we unexpectedly found the limb preference of rodent PPC neurons apparently biased to the ipsilateral side in the present study. This seems paradoxical in that the cerebral cortex, in principle, governs the sensory and motor system contralaterally according to the conventional theory established in neuroscience.

How can we understand the paradoxically ipsilaterally biased limb preference of rodent PPC neurons? It is well known that the PPC has axonal connections with sensory cortices and participates in a variety of cognitive functions (e.g., sensory history: Akrami et al., 2018 or evidence accumulation: Erlich et al., 2015; Scott et al., 2017). Therefore, our optogenetic PPC activation could give some perturbation to a sensory feedback for cognitive function on the contralateral side, resulting in a compensatory appearance of ipsilateral movements. However, this is unlikely because the ipsilateral preference was observed even in PPC activity during natural task performance without cortical activation/inhibition. Also, such cognitive information is usually processed in both hemispheres (Li et al., 2016).

Now consider the case when a rat is releasing the right pedal with his right forelimb. The left M1 neurons would shape an actual motor command (force, direction, etc.) to execute muscle contractions to extend or flex the right (contralateral) forelimb, for example, releasing the pedal with the right forelimb. The left M2 neurons would, in advance, process motor information to organize multiple limb movements, for example, releasing with the right forelimb and holding with the left forelimb (Soma et al., 2017), similarly to the PM/SMA neurons in primates (Tanji et al., 1987; Cisek et al., 2003; Kurata, 2010; Nakayama et al., 2015). Therefore, M1 and M2 neurons participate in controlling any form of movements of forelimb(s) including extension and flexion. M2 neurons have reciprocal connections with those on the other side (Shepherd, 2013) and receive axonal inputs from PPC projection neurons (Corwin and Reep, 1998). Upstream PPC neurons in both hemispheres might then coordinate more abstract motor information on a motor pattern of movements of multiple limbs. In general, rodents use their forelimbs as front 
legs for locomotion such as walking and running (Chen et al., 1994; McNaughton et al., 1994; Nitz, 2006; Kitsukawa et al., 2011; Whitlock et al., 2012; Soma et al., 2014, 2018; Wilber et al., 2014a), unlike primate forelimbs, which are used skillfully for discrete movements such as reaching and grasping (Tanji et al., 1987; Franz, 2003). Therefore, the motor pattern involving PPC neurons in rodents should be naturally associated with their locomotion with four limbs, although the PPC just modulates the locomotion driven by its central pattern generator in the spinal cord (Drew and Marigold, 2015). In each cycle of locomotion, a stance against the ground will require stronger activation of antigravity muscles than a swing of the limb. Supposing that the right PPC neurons preferentially encode a basic pattern of stance with the left limb and swing with the right limb for locomotion, then they would represent an abstract (not actual) requirement of larger force for the left stance than the right swing, which corresponds to the contralaterally biased preference of M1 and M2 neurons. This pattern, regardless of actual force, can be applied to the right pedal release (corresponding to swing) and the left pedal hold (stance) in the behavioral task condition. Using the motor pattern from PPC, the M1 and M2 neurons might process actual information on force and direction for the planning/preparation and execution of forelimb movements. Although this is just speculation, it explains the apparent preference of PPC neurons for the ipsilateral forelimb as well as the swing-like movements of the ipsilateral forelimb after PPC activation. Therefore, we would assume that the function of the rodent PPC is likely to be optimized for quadrupedal locomotion. In fact, cat PPC neurons are known to increase their activity just before the animal strides over an obstacle with the forelimbs during locomotion (Drew and Marigold, 2015; Marigold and Drew, 2017). Similarly, the function of the primate PPC appears to be highly optimized for skilled forelimb movements such as reaching and grasping (Taira et al., 1990; Gallese et al., 1994; Sakata et al., 1995; Desmurget et al., 1999; Connolly et al., 2003). Using the results of this study and studies in other mammals, it is possible that the evolution from quadrupedal to bipedal-like mammals may explain the functional differentiation of the parietal association cortex.

\section{Possible circuit mechanism underlying ipsilateral limb preference in rodent PPC neurons}

Apart from our motor pattern hypothesis, what is the circuit mechanism of ipsilateral limb preference in PPC neurons? One possibility is that ipsilateral PPC neurons drive motor cortex neurons contralaterally, eventually leading to ipsilateral forelimb movements. However, this seems unlikely because cortical projection neurons send their axons contralaterally to the counterpart area as well as ipsilaterally to different cortical areas, but only weakly to other contralateral areas (Yamawaki and Shepherd, 2015; Yamawaki et al., 2016). Therefore, it is unlikely that the PPC neurons send motor information directly to motor cortex neurons contralaterally. Another possibility is that PPC activation per se may affect descending subcortical signals through extrapyramidal tract pathways; for example, an indirect inactivation (releasing from reflex maintenance) of antigravity muscles in ipsilateral forelimb via the medullary reticulospinal tract (Bear et al., 2007).

Our current experiments have generated new questions about functional PPC circuitry. In our spike analysis, both RS and FS subtypes of PPC neurons (putative pyramidal cells and inhibitory interneurons, respectively) showed more or less ipsilaterally biased limb preference (Fig. 5). However, it remains unclear what types of pyramidal cells (e.g., intratelencephalic or pyramidal tract neurons; Soma et al., 2017; Baker et al., 2018; Ebbesen et al., 2018; Isomura, 2018; Saiki et al., 2018) or versatile interneurons actually contribute to controlling the ipsilateral forelimb movements as elements of the PPC circuit. We also demonstrated that ipsilateral forelimb movements were evoked optogenetically in the whole part of PPC (Fig. 8), but we did not map evoked movements of other body parts separately. Therefore, whether somatotopy exists in the PPC is unresolved. Optogenetic identification/manipulation using more specific transgenic animals and more precise optical stimulation (Harrison et al., 2012; Hira et al., 2015) will help us to answer these questions about the functional PPC circuitry for motor control in the future.

\section{References}

Akrami A, Kopec CD, Diamond ME, Brody CD (2018) Posterior parietal cortex represents sensory history and mediates its effects on behaviour. Nature 554:368-372. CrossRef Medline

Baker A, Kalmbach B, Morishima M, Kim J, Juavinett A, Li N, Dembrow N (2018) Specialized subpopulations of deep-layer pyramidal neurons in the neocortex: bridging cellular properties to functional consequences. J Neurosci 38:5441-5455. CrossRef Medline

Baldwin MK, Cooke DF, Krubitzer L (2017) Intracortical microstimulation maps of motor, somatosensory, and posterior parietal cortex in tree shrews (Tupaia belangeri) reveal complex movement representations. Cereb Cortex 27:1439-1456. CrossRef Medline

Bear MF, Connors BW, Paradiso MA (2007) The pontine and medullary reticulospinal tracts. In: Neuroscience: exploring the brain (Bear MF, Connors BW, Paradiso MA, eds), pp 457-458. Baltimore: Lippincott Williams and Wilkins.

Chandler HC, King V, Corwin JV, Reep RL (1992) Thalamocortical connections of rat posterior parietal cortex. Neurosci Lett 143:237-242. CrossRef Medline

Chang SW, Snyder LH (2012) The representations of reach endpoints in posterior parietal cortex depend on which hand does the reaching. J Neurophysiol 107:2352-2365. CrossRef Medline

Chang SW, Dickinson AR, Snyder LH (2008) Limb-specific representation for reaching in the posterior parietal cortex. J Neurosci 28:6128-6140. CrossRef Medline

Chen LL, Lin LH, Barnes CA, McNaughton BL (1994) Head-direction cells in the rat posterior cortex. II. Contributions of visual and ideothetic information to the directional firing. Exp Brain Res 101:24-34. CrossRef Medline

Churchland MM, Yu BM, Ryu SI, Santhanam G, Shenoy KV (2006) Neural variability in premotor cortex provides a signature of motor preparation. J Neurosci 26:3697-3712. CrossRef Medline

Cisek P, Crammond DJ, Kalaska JF (2003) Neural activity in primary motor and dorsal premotor cortex in reaching tasks with the contralateral versus ipsilateral arm. J Neurophysiol 89:922-942. CrossRef Medline

Connolly JD, Andersen RA, Goodale MA (2003) FMRI evidence for a 'parietal reach region' in the human brain. Exp Brain Res 153:140-145. CrossRef Medline

Corwin JV, Reep RL (1998) Rodent posterior parietal cortex as a component of a cortical network mediating directed spatial attention. Psychobiology 26:87-102.

Deffeyes JE, Touvykine B, Quessy S, Dancause N (2015) Interactions between rostral and caudal cortical motor areas in the rat. J Neurophysiol 113:3893-3904. CrossRef Medline

Desmurget M, Epstein CM, Turner RS, Prablanc C, Alexander GE, Grafton ST (1999) Role of the posterior parietal cortex in updating reaching movements to a visual target. Nat Neurosci 2:563-567. CrossRef Medline

Donoghue JP, Wise SP (1982) The motor cortex of the rat: cytoarchitecture and microstimulation mapping. J Comp Neurol 212:76-88. CrossRef Medline

Drew T, Marigold DS (2015) Taking the next step: cortical contributions to the control of locomotion. Curr Opin Neurobiol 33:25-33. CrossRef Medline

Ebbesen CL, Insanally MN, Kopec CD, Murakami M, Saiki A, Erlich JC (2018) More than just a "motor": recent surprises from the frontal cortex. J Neurosci 38:9402-9413. CrossRef Medline

Erlich JC, Brunton BW, Duan CA, Hanks TD, Brody CD (2015) Distinct 
effects of prefrontal and parietal cortex inactivations on an accumulation of evidence task in the rat. eLife 4:e05457. CrossRef Medline

Evarts EV (1966) Pyramidal tract activity associated with a conditioned hand movement in the monkey. J Neurophysiol 29:1011-1027. CrossRef Medline

Franz EA (2003) Bimanual action representation: a window to human evolution. In: Taking action: cognitive neuroscience perspectives on intentional acts. (Johnson-Frey SH, ed), pp 259-288. Cambridge, MA: MIT.

Gallese V, Murata A, Kaseda M, Niki N, Sakata H (1994) Deficit of hand preshaping after muscimol injection in monkey parietal cortex. Neuroreport 5:1525-1529. CrossRef Medline

Gharbawie OA, Stepniewska I, Kaas JH (2011a) Cortical connections of functional zones in posterior parietal cortex and frontal cortex motor regions in new world monkeys. Cereb Cortex 21:1981-2002. CrossRef Medline

Gharbawie OA, Stepniewska I, Qi H, Kaas JH (2011b) Multiple parietalfrontal pathways mediate grasping in macaque monkeys. J Neurosci 31: 11660-11677. CrossRef Medline

Goard MJ, Pho GN, Woodson J, Sur M (2016) Distinct roles of visual, parietal, and frontal motor cortices in memory-guided sensorimotor decisions. eLife 5:e13764. CrossRef Medline

Griffin DM, Hoffman DS, Strick PL (2015) Corticomotoneuronal cells are "functionally tuned". Science 350:667-670. CrossRef Medline

Hanks TD, Kopec CD, Brunton BW, Duan CA, Erlich JC, Brody CD (2015) Distinct relationships of parietal and prefrontal cortices to evidence accumulation. Nature 520:220-223. CrossRef Medline

Harrison TC, Ayling OG, Murphy TH (2012) Distinct cortical circuit mechanisms for complex forelimb movement and motor map topography. Neuron 74:397-409. CrossRef Medline

Hazan L, Zugaro M, Buzsáki G (2006) Klusters, NeuroScope, NDManager: a free software suite for neurophysiological data processing and visualization. J Neurosci Methods 155:207-216. CrossRef Medline

Hira R, Terada S, Kondo M, Matsuzaki M (2015) Distinct functional modules for discrete and rhythmic forelimb movements in the mouse motor cortex. J Neurosci 35:13311-13322. CrossRef Medline

Hoshi E, Tanji J (2000) Integration of target and body-part information in the premotor cortex when planning action. Nature 408:466-470. CrossRef Medline

Igarashi H, Koizumi K, Kaneko R, Ikeda K, Egawa R, Yanagawa Y, Muramatsu S, Onimaru H, Ishizuka T, Yawo H (2016) A novel reporter rat strain that conditionally expresses the bright red fluorescent protein tdTomato. PLoS One 11:e0155687. CrossRef Medline

Igarashi H, Ikeda K, Onimaru H, Kaneko R, Koizumi K, Beppu K, Nishizawa K, Takahashi Y, Kato F, Matsui K, Kobayashi K, Yanagawa Y, Muramatsu SI, Ishizuka T, Yawo H (2018) Targeted expression of step-function opsins in transgenic rats for optogenetic studies. Sci Rep 8:5435. CrossRef Medline

Isomura Y (2018) Multi-Linc: a new approach for exploring inter-areal spike communication. In: Advances in cognitive neurodynamics (VI) (Delgado-García JM, Pan X, Sánchez-Campusano R, Wang R, ed), pp 189-193. Heidelberg: Springer.

Isomura Y, Harukuni R, Takekawa T, Aizawa H, Fukai T (2009) Microcircuitry coordination of cortical motor information in self-initiation of voluntary movements. Nat Neurosci 12:1586-1593. CrossRef Medline

Kaas JH, Stepniewska I (2016) Evolution of posterior parietal cortex and parietal-frontal networks for specific actions in primates. J Comp Neurol 524:595-608. CrossRef Medline

Kermadi I, Liu Y, Rouiller EM (2000) Do bimanual motor actions involve the dorsal premotor $(\mathrm{PMd})$, cingulate $(\mathrm{CMA})$ and posterior parietal (PPC) cortices? Comparison with primary and supplementary motor cortical areas. Somatosens Mot Res 17:255-271. CrossRef Medline

Kimura R, Saiki A, Fujiwara-Tsukamoto Y, Sakai Y, Isomura Y (2017) Large-scale analysis reveals populational contributions of cortical spike rate and synchrony to behavioural functions. J Physiol 595:385-413. CrossRef Medline

Kitsukawa T, Nagata M, Yanagihara D, Tomioka R, Utsumi H, Kubota Y, Yagi T, Graybiel AM, Yamamori T (2011) A novel instrumented multipeg running wheel system, Step-Wheel, for monitoring and controlling complex sequential stepping in mice. J Neurophysiol 106:479-487. CrossRef Medline

Kurata K (2007) Laterality of movement-related activity reflects transfor- mation of coordinates in ventral premotor cortex and primary motor cortex of monkeys. J Neurophysiol 98:2008-2021. CrossRef Medline

Kurata K (2010) Conditional selection of contralateral and ipsilateral forelimb movements by the dorsal premotor cortex in monkeys. J Neurophysiol 103:262-277. CrossRef Medline

Li N, Daie K, Svoboda K, Druckmann S (2016) Robust neuronal dynamics in premotor cortex during motor planning. Nature 532:459-464. CrossRef Medline

Marigold DS, Drew T (2017) Posterior parietal cortex estimates the relationship between object and body location during locomotion. eLife 6:e28143. CrossRef Medline

McNaughton BL, Mizumori SJ, Barnes CA, Leonard BJ, Marquis M, Green EJ (1994) Cortical representation of motion during unrestrained spatial navigation in the rat. Cereb Cortex 4:27-39. CrossRef Medline

Mushiake H, Inase M, Tanji J (1991) Neuronal activity in the primate premotor, supplementary, and precentral motor cortex during visually guided and internally determined sequential movements. J Neurophysiol 66:705-718. CrossRef Medline

Nakayama Y, Yokoyama O, Hoshi E (2015) Distinct neuronal organizations of the caudal cingulate motor area and supplementary motor area in monkeys for ipsilateral and contralateral hand movements. J Neurophysiol 113:2845-2858. CrossRef Medline

Narayanan NS, Laubach M (2009) Delay activity in rodent frontal cortex during a simple reaction time task. J Neurophysiol 101:2859-2871. CrossRef Medline

Neafsey EJ, Bold EL, Haas G, Hurley-Gius KM, Quirk G, Sievert CF, Terreberry RR (1986) The organization of the rat motor cortex: a microstimulation mapping study. Brain Res 11:77-96. CrossRef Medline

Nitz DA (2006) Tracking route progression in the posterior parietal cortex. Neuron 49:747-756. CrossRef Medline

Nonomura S, Nishizawa K, Sakai Y, Kawaguchi Y, Kato S, Uchigashima M, Watanabe M, Yamanaka K, Enomoto K, Chiken S, Sano H, Soma S, Yoshida J, Samejima K, Ogawa M, Kobayashi K, Nambu A, Isomura Y, Kimura M (2018) Monitoring and updating of action selection for goaldirected behavior through the striatal direct and indirect pathways. Neuron 99:1302-1314.e5. CrossRef Medline

Olsen GM, Witter MP (2016) Posterior parietal cortex of the rat: architectural delineation and thalamic differentiation. J Comp Neurol 524:37743809. CrossRef Medline

Powell K, Mathy A, Duguid I, Häusser M (2015) Synaptic representation of locomotion in single cerebellar granule cells. eLife 4:e07290. CrossRef Medline

Quian Quiroga R, Snyder LH, Batista AP, Cui H, Andersen RA (2006) Movement intention is better predicted than attention in the posterior parietal cortex. J Neurosci 26:3615-3620. CrossRef Medline

Saiki A, Kimura R, Samura T, Fujiwara-Tsukamoto Y, Sakai Y, Isomura Y (2014) Different modulation of common motor information in rat primary and secondary motor cortices. PLoS One 9:e98662. CrossRef Medline

Saiki A, Sakai Y, Fukabori R, Soma S, Yoshida J, Kawabata M, Yawo H, Kobayashi K, Kimura M, Isomura Y (2018) In vivo spiking dynamics of intra-and extratelencephalic projection neurons in rat motor cortex. Cereb Cortex 28:1024-1038. CrossRef Medline

Sakata H, Taira M, Murata A, Mine S (1995) Neural mechanisms of visual guidance of hand action in the parietal cortex of the monkey. Cereb Cortex 5:429-438. CrossRef Medline

Scott BB, Constantinople CM, Akrami A, Hanks TD, Brody CD, Tank DW (2017) Fronto-parietal cortical circuits encode accumulated evidence with a diversity of timescales. Neuron 95:385-398.e5. CrossRef Medline

Shadlen MN, Newsome WT (2001) Neural basis of a perceptual decision in the parietal cortex (area LIP) of the rhesus monkey. J Neurophysiol 86: 1916-1936. CrossRef Medline

Shenoy KV, Sahani M, Churchland MM (2013) Cortical control of arm movements: a dynamical systems perspective. Annu Rev Neurosci 36: 337-359. CrossRef Medline

Shepherd GM (2013) Corticostriatal connectivity and its role in disease. Nat Rev Neurosci 14:278-291. CrossRef Medline

Snyder LH, Batista AP, Andersen RA (1997) Coding of intention in the posterior parietal cortex. Nature 386:167-170. CrossRef Medline

Snyder LH, Batista AP, Andersen RA (1998) Change in motor plan, without a change in the spatial locus of attention, modulates activity in posterior parietal cortex. J Neurophysiol 79:2814-2819. CrossRef Medline 
Snyder LH, Batista AP, Andersen RA (2000) Intention-related activity in the posterior parietal cortex: a review. Vis Res 40:1433-1441. CrossRef Medline

Soma S, Shimegi S, Osaki H, Sato H (2012) Cholinergic modulation of response gain in the primary visual cortex of the macaque. J Neurophysiol 107:283-291. CrossRef Medline

Soma S, Suematsu N, Shimegi S (2014) Efficient training protocol for rapid learning of the two-alternative forced-choice visual stimulus detection task. Physiol Rep 2:e12060. CrossRef Medline

Soma S, Saiki A, Yoshida J, Ríos A, Kawabata M, Sakai Y, Isomura Y (2017) Distinct laterality in forelimb-movement representations of rat primary and secondary motor cortical neurons with intratelencephalic and pyramidal tract projections. J Neurosci 37:10904-10916. CrossRef Medline

Soma S, Suematsu N, Yoshida J, Ríos A, Shimegi S (2018) Discretion for behavioral selection affects development of habit formation after extended training in rats. Behav Processes 157:291-300. CrossRef Medline

Stark A, Zohary E (2008) Parietal mapping of visuomotor transformations during human tool grasping. Cereb Cortex 18:2358-2368. CrossRef Medline

Stepniewska I, Fang PC, Kaas JH (2005) Microstimulation reveals specialized subregions for different complex movements in posterior parietal cortex of prosimian galagos. Proc Natl Acad Sci U S A 102:4878-4883. CrossRef Medline

Stepniewska I, Fang PC, Kaas JH (2009) Organization of the posterior parietal cortex in galagos: I. functional zones identified by microstimulation. J Comp Neurol 517:765-782. CrossRef Medline

Sugimura YK, Takahashi Y, Watabe AM, Kato F (2016) Synaptic and network consequences of monosynaptic nociceptive inputs of parabrachial nucleus origin in the central amygdala. J Neurophysiol 115:2721-2739. CrossRef Medline

Taira M, Mine S, Georgopoulos AP, Murata A, Sakata H (1990) Parietal cortex neurons of the monkey related to the visual guidance of hand movement. Exp Brain Res 83:29-36. Medline

Takekawa T, Isomura Y, Fukai T (2010) Accurate spike sorting for multiunit recordings. Eur J Neurosci 31:263-272. CrossRef Medline
Takekawa T, Isomura Y, Fukai T (2012) Spike sorting of heterogeneous neuron types by multimodality-weighted PCA and explicit robust variational bayes. Front Neuroinform 6:5. CrossRef Medline

Tanji J, Okano K, Sato KC (1987) Relation of neurons in the nonprimary motor cortex to bilateral hand movement. Nature 327:618-620. CrossRef Medline

Tomita H, Sugano E, Fukazawa Y, Isago H, Sugiyama Y, Hiroi T, Ishizuka T, Mushiake H, Kato M, Hirabayashi M, Shigemoto R, Yawo H, Tamai M (2009) Visual properties of transgenic rats harboring the channelrhodopsin-2 gene regulated by the thy- 1.2 promoter. PLoS One 4:e7679. CrossRef Medline

Weinrich M, Wise SP (1982) The premotor cortex of the monkey. J Neurosci 2:1329-1345. CrossRef Medline

Whitlock JR, Sutherland RJ, Witter MP, Moser MB, Moser EI (2008) Navigating from hippocampus to parietal cortex. Proc Natl Acad Sci U S A 105:14755-14762. CrossRef Medline

Whitlock JR, Pfuhl G, Dagslott N, Moser MB, Moser EI (2012) Functional split between parietal and entorhinal cortices in the rat. Neuron 73:789802. CrossRef Medline

Wilber AA, Clark BJ, Forster TC, Tatsuno M, McNaughton BL (2014a) Interaction of egocentric and world-centered reference frames in the rat posterior parietal cortex. J Neurosci 34:5431-5446. CrossRef Medline

Wilber AA, Clark BJ, Demecha AJ, Mesina L, Vos JM, McNaughton BL (2014b) Cortical connectivity maps reveal anatomically distinct areas in the parietal cortex of the rat. Front Neural Circuits 8:146. CrossRef Medline

Yamawaki N, Shepherd GM (2015) Synaptic circuit organization of motor corticothalamic neurons. J Neurosci 35:2293-2307. CrossRef Medline

Yamawaki N, Radulovic J, Shepherd GM (2016) A corticocortical circuit directly links retrosplenial cortex to M2 in the mouse. J Neurosci 36:93659374. CrossRef Medline

Yoshida J, Saiki A, Soma S, Yamanaka K, Nonomura S, Ríos A, Kawabata M, Kimura M, Sakai Y, Isomura Y (2018) Area-specific modulation of functional cortical activity during block-based and trial-based proactive inhibition. Neuroscience 388:297-316. CrossRef Medline 